\title{
Asymptotic properties of random unlabelled block-weighted graphs
}

\author{
Benedikt Stufler \\ Vienna University of Technology \\ Institute of Discrete Mathematics and Geometry \\ Vienna, Austria \\ benedikt.stufler [at] tuwien.ac.at
}

Submitted: Oct 5, 2020; Accepted: Oct 12, 2021; Published: Nov 19, 2021

(C) The authors. Released under the CC BY-ND license (International 4.0).

\begin{abstract}
We study the asymptotic shape of random unlabelled graphs subject to certain subcriticality conditions. The graphs are sampled with probability proportional to a product of Boltzmann weights assigned to their 2-connected components. As their number of vertices tends to infinity, we show that they admit the Brownian tree as Gromov-Hausdorff-Prokhorov scaling limit, and converge in a strengthened Benjamini-Schramm sense toward an infinite random graph. We also consider models of random graphs that are allowed to be disconnected. Here a giant connected component emerges and the small fragments converge without any rescaling towards a finite random limit graph.
\end{abstract}

Mathematics Subject Classifications: 60C05, 05C80

\section{Introduction}

\subsection{General context and motivation}

The study of probabilistic and combinatorial properties of graphs from restricted classes has received increasing attention in recent literature. Prominent examples of such classes include trees [22], outerplanar graphs [9, 37], planar graphs [35], and general families like subcritical graph classes $[16,15,17]$ and bridge-addable graph classes [13]. A fundamental problem in this context is to describe the asymptotic shape of a random graph from such a class as its number of vertices tends to infinity. For example, we could ask "what is the structure of a typical large planar graph"? This question actually concerns two canonical models of random graphs. First, we could uniformly pick a graph among all planar graphs with a given $n$-element vertex set. Second, we could uniformly pick a graph among all unlabelled planar graphs with $n$ vertices. The two models are not equivalent. Picking a 
labelled graph creates a bias that is related to the size of the automorphism group. This is strongly visible from an enumerative standpoint as well. For example, the number of labelled and unlabelled trees admit different asymptotic expressions [29]. Enumerating and studying unlabelled graphs also poses particular challenges. For example, the asymptotic number of labelled planar graphs has been determined in breakthrough work by Giménez and Noy [21], concluding a long history of rough estimates. Determining the asymptotic number and shape of unlabelled planar graphs, however, still remains an open problem.

This strongly motivates the development of methods that allow us to understand the typical structure of graph symmetries. An important addition to this toolbox was made in recent work by Bodirsky, Fusy, Kang, and Vigerske [10], who introduced the method of cycle pointing. Roughly speaking, instead of using the common enumerative method of rooting graphs at vertices, they are rooted at cycles of automorphisms. As demonstrated in [10], cycle pointing allows also for the construction of Boltzmann samplers for the efficient generation of random unlabelled objects. These samplers open doors to a probabilistic analysis. The already quite demanding special case of unlabelled trees has been studied in this way in [34], but it is desirable to obtain results for graphs that are not necessarily trees.

\subsection{Main result}

In the present work, we use cycle pointing to study a general model of random tree-like unlabelled graphs. Suppose that for each 2-connected graph $B$ (including the complete graph $K_{2}$ with two vertices) we are given a weight $\iota(B) \geqslant 0$ such that isomorphic graphs receive the same weight. To any connected graph $C$ we may then assign the weight

$$
\omega(C)=\prod_{B} \iota(B)
$$

with the index $B$ ranging over all 2-connected components of $C$, that is, maximal 2connected subgraphs. If $C$ consists of a single vertex, then it receives weight 1 . We may then consider the random connected unlabelled graph $\mathrm{C}_{n}^{\omega}$ with $n$ vertices, sampled with probability proportional to its $\omega$-weight, and likewise the random connected unlabelled rooted graph $\mathrm{A}_{n}^{\omega}$. Drawing a random graph according to such block-weights encompasses random unlabelled graphs from block-stable classes, which correspond to the special case where each $\iota$-weight is required to be either equal to 1 or 0 . Block-stable classes of graphs have received attention in recent literature, see McDiarmid and Scott [27].

The direct study of $\mathrm{C}_{n}^{\omega}$ is challenging, as the structure of the symmetries of objects without roots is much more complex as in the rooted case, where each symmetry is required to fix the root vertex. So, instead of directly studying unrooted unlabelled graphs, we are going to take a more economic approach and geometrically approximate $\mathrm{C}_{n}^{\omega}$ by a random rooted graph having size $n+O_{p}(1)$. More precisely, we are going to construct a random rooted graph $\mathrm{D}_{n}$ with size $d_{n}=\left|\mathrm{D}_{n}\right|=O_{p}(1)$ such that the graph $\mathrm{D}_{n}+\mathrm{A}_{n-d_{n}}^{\omega}$ obtained by gluing the root of $\mathrm{D}_{n}$ to the root of $\mathrm{A}_{n-h_{n}}^{\omega}$ approximates $\mathrm{C}_{n}^{\omega}$ in total variation. That is, $\mathrm{D}_{n}+\mathrm{A}_{n-d_{n}}^{\omega}$ is formed by identifying the root vertices of the graphs $\mathrm{D}_{n}$ and $\mathrm{A}_{n-d_{n}}^{\omega}$. 
In order for the random graph $\mathrm{C}_{n}^{\omega}$ to behave in a tree-like manner, we will make an assumption on the weight-sequence, which generalizes the definition of subcriticality for block-stable classes of unlabelled graphs given in [15, Sec. 5]: Define the cycle index sum

$$
Z_{\left(\mathscr{B}^{\prime}\right)^{\iota}}\left(s_{1}, s_{2}, \ldots\right)=\sum_{k \geqslant 1} \sum_{B^{\prime} \in \mathscr{B}_{k}^{\prime}} \frac{\iota\left(B^{\prime}\right)}{k !} \sum_{\sigma} s_{1}^{\sigma_{1}} s_{2}^{\sigma_{2}} \cdots s_{k}^{\sigma_{k}},
$$

with $\mathscr{B}_{k}^{\prime}$ denoting the set of all 2 -connected graphs with vertex set $\{*, 1, \ldots, k\}$ for arbitrary $k \geqslant 1$, the sum index $\sigma:[k] \rightarrow[k]$ ranging over the elements of the permutation group of order $k$ such that the canonically extension $\bar{\sigma}$ with $\left.\bar{\sigma}\right|_{[k]}=\sigma$ and $\bar{\sigma}(*)=*$ is an automorphism of $B^{\prime}$, and $\sigma_{i}$ denoting the number of cycles of length $i$ in $\sigma$. (The reason for the prime in $\mathscr{B}^{\prime}$ is that this is an instance of a derived species, see Appendix $\mathrm{A}$ for more details on the symbolic method.) Likewise, we define the cycle sum

$$
Z_{(\mathscr{C}) \omega}\left(s_{1}, s_{2}, \ldots\right)=\sum_{k \geqslant 1} \sum_{C \in \mathscr{C}_{k}} \frac{\omega(C)}{k !} \sum_{\sigma} s_{1}^{\sigma_{1}} s_{2}^{\sigma_{2}} \cdots s_{k}^{\sigma_{k}}
$$

with $\mathscr{C}_{k}$ denoting the set of all connected graphs with labels in $[k]$ and the sum index $\sigma$ ranging over automorphisms of $C$. Furthermore, we set for each $i \geqslant 1$

$$
\tilde{\mathscr{A}}^{\omega^{i}}(z)=\sum_{A} \omega(A)^{i} z^{|A|}
$$

with the sum index $A$ ranging over all rooted unlabelled graphs. (The tilde in $\tilde{\mathscr{A}}$ indicates that this is an ordinary generating series, as opposed to an exponential generating series. We refer to Appendix A for relevant background on these notions.) We require that the radius of convergence $\rho_{\mathscr{A}}$ of $\mathscr{A}^{\omega}(z)$ and the bivariate sum

$$
g(x, y)=\exp \left(Z_{\left(\mathscr{B}^{\prime}\right)^{\iota}}\left(x, \tilde{\mathscr{A}}^{\omega^{2}}\left(y^{2}\right), \tilde{\mathscr{A}}^{\omega^{3}}\left(y^{3}\right), \ldots\right)+\sum_{i \geqslant 2} \frac{1}{i} Z_{\left(\mathscr{B}^{\prime}\right)^{\iota}}\left(\tilde{\mathscr{A}}^{\omega^{i}}\left(y^{i}\right), \tilde{\mathscr{A}}^{\omega^{2 i}}\left(y^{2 i}\right), \ldots\right)\right)
$$

satisfy

$$
\rho_{\mathscr{A}}>0, \quad g\left(\tilde{\mathscr{A}}^{\omega}\left(\rho_{\mathscr{A}}\right)+\epsilon, \rho_{\mathscr{A}}+\epsilon\right)<\infty, \quad \text { and } \quad Z_{\mathscr{C} \omega}\left(0,\left(\rho_{\mathscr{A}}+\epsilon\right)^{2},\left(\rho_{\mathscr{A}}+\epsilon\right)^{3}, \ldots\right)<\infty
$$

for some $\epsilon>0$.

Although this Assumption seems rather abstract, it is known to be satisfied for a wide range of random graphs that appear naturally in combinatorics. For example, condition (2) holds if $C_{n}^{\omega}$ is the uniform random connected unlabelled series-parallel graph, cacti graph or outerplanar graph with $n$ vertices. Or, more generally, this encompasses random graphs from subcritical classes of unlabelled graphs, which include random graphs from classes defined by a finite set of 3-connected components. We refer the reader to work by Drmota, Fusy, Kang, Kraus, and Rué [15, Sec. 6] for details. A famous class for which condition (2) fails is the class of unlabelled planar graphs. 
Theorem 1. Suppose that Assumption (2) is satisfied. Then there exists a coupling of $\mathbf{C}_{n}^{\omega}$ with a random rooted graph $\mathrm{D}_{n}$ with size $d_{n}=\left|\mathrm{D}_{n}\right|=O_{p}(1)$, and the random rooted graph $\mathrm{A}_{n-h_{n}}^{\omega}$, such that the graph $\mathrm{D}_{n}+\mathrm{A}_{n-h_{n}}^{\omega}$ obtained by identifying the root of $\mathrm{D}_{n}$ with the root of $\mathrm{A}_{n-h_{n}}^{\omega}$ approximates $\mathrm{C}_{n}^{\omega}$ in total variation. The speed of convergence is exponential, that is, there exist constants $C, c>0$ that do not depend on $n$ such that

$$
d_{\mathrm{TV}}\left(\mathrm{C}_{n}^{\omega}, \mathrm{D}_{n}+\mathrm{A}_{n-d_{n}}^{\omega}\right) \leqslant C \exp (-c n)
$$

for all $n$.

Theorem 1 allows us to transfer a large class of asymptotic graph properties from $A_{n}^{\omega}$ to $C_{n}^{\omega}$. Note that this approach does not work as well the other way. We may at best deduce that an asymptotic property of $\mathrm{C}_{n}^{\omega}$ must also hold for the random rooted graph $\mathrm{A}_{n-h_{n}}^{\omega}$ which has a random size, but this does a priori not imply that the property also asymptotically holds for $\mathrm{A}_{n}^{\omega}$ which has a deterministic size.

\subsection{Scaling limit and consequences}

We now state some important consequences of Theorem 1. The following theorem states that the asymptotic shape of the random graphs under consideration is tree-like. Compare with Figure 2 which illustrates a large randomly generated cactus graph.

Theorem 2. Suppose that (2) holds, and let $\mu_{n}$ denote the uniform measure on the vertices of $\mathrm{C}_{n}^{\omega}$. Then there exists a constant $c_{\omega}>0$ such that

$$
\left(\mathrm{C}_{n}^{\omega}, \frac{c_{\omega}}{\sqrt{n}} d_{\mathrm{C}_{n}^{\omega}}, \mu_{n}\right) \stackrel{d}{\longrightarrow}\left(\mathcal{T}_{\mathrm{e}}, d_{\mathcal{T}_{\mathrm{e}}}, \mu\right)
$$

in the Gromov-Hausdorff-Prokhorov sense, with $\left(\mathcal{T}_{\mathrm{e}}, d_{\mathcal{T}_{\mathrm{e}}}, \mu\right)$ denoting the Brownian continuum random tree. Moreover, there are constants $C, c>0$ such the diameter $\mathbf{D}\left(\mathrm{C}_{n}^{\omega}\right)$ satisfies for all $n$ the tail bound

$$
\mathbb{P}\left(\mathbf{D}\left(\mathrm{C}_{n}^{\omega}\right) \geqslant x\right) \leqslant C \exp \left(-c x^{2} / n\right)
$$

The Brownian continuum random tree consists of a random compact metric space $\left(\mathcal{T}_{\mathrm{e}}, d_{\mathcal{T}_{\mathrm{e}}}\right)$ together with a probability measure $\mu$ on its Borel $\sigma$-algebra. The letter e $=$ $\left(\mathrm{e}_{t}\right)_{0 \leqslant t \leqslant 1}$ denotes a Brownian excursion normalized to have duration 1. Briefly summarized, $\left(\mathcal{T}_{\mathrm{e}}, d_{\mathcal{T}_{\mathrm{e}}}\right)$ may be defined as the quotient metric space corresponding to the random pre-metric

$$
d_{\mathrm{e}}(x, y)=\mathrm{e}_{\min (x, y)}+\mathrm{e}_{\max (x, y)}-2 \inf _{\min (x, y) \leqslant t \leqslant \max (x, y)} \mathrm{e}_{t}
$$

on the compact unit interval $[0,1]$. The random probability measure $\mu$ is the push-forward measure of the Lebesgue measure on $[0,1]$. We refer to $[3,4,5]$ for details on the Brownian continuum random tree, and to [28, Sec. 6] for details on Gromov-Hausdorff-Prokhorov 


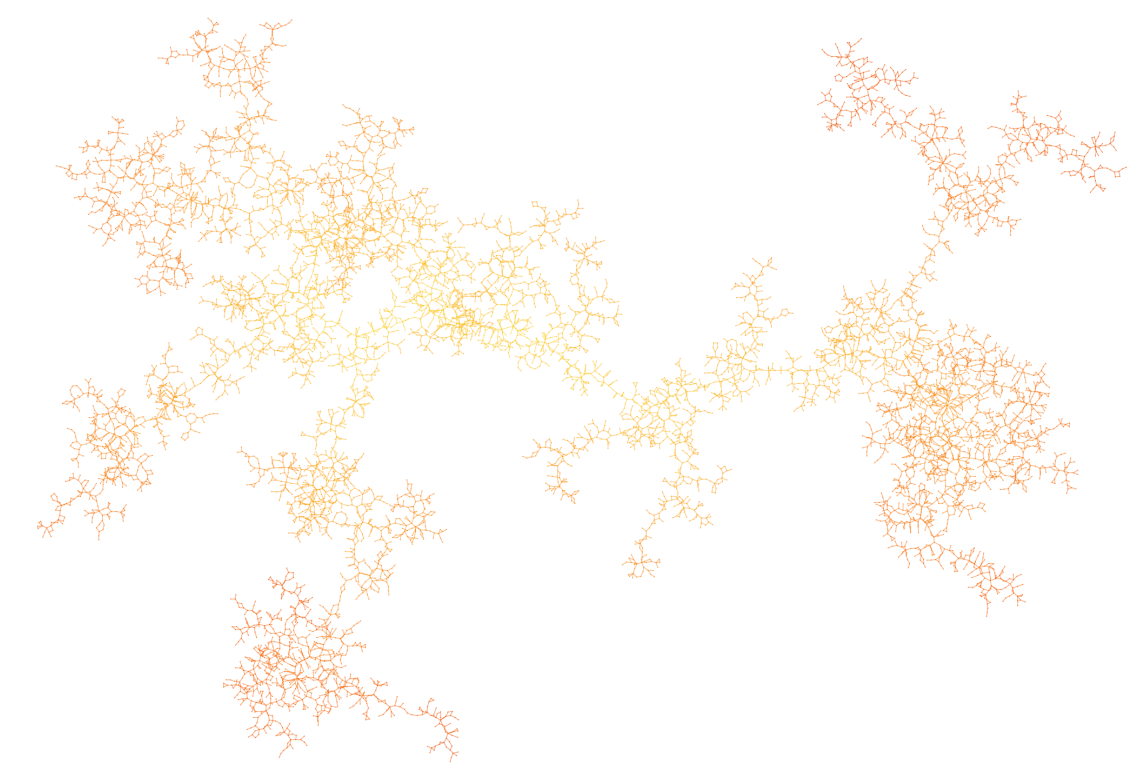

Figure 1: A random cactus graph with 10k vertices which are coloured according to their closeness centrality.

convergence of random compact measured metric spaces. See also Section 5.1 below for a concise introduction.

Theorem 2 recovers the scaling limit for the model of uniform random unlabelled trees without degree restrictions by [34]. We do not recover the results for degree restricted unlabelled trees from [34], as treating general graphs forces us to take different paths in the cycle decomposition.

The idea behind the scaling limit of $\mathrm{C}_{n}^{\omega}$ is that the graph $\mathrm{D}_{n}$ contracts to a single point when rescaled by $n^{-1 / 2}$, and hence the Gromov-Hausdorff-Prokhorov distance between the rescaled versions of $A_{n-h_{n}}^{\omega}$ and $C_{n}^{\omega}$ tends in probability to zero. Hence we may build upon previous scaling limits for the rooted graph $A_{n}^{\omega}$ given in [33, Thm. 33].

Theorem 2 has various applications. For example, it follows from (4) that

$$
c_{\omega} \mathbf{D}\left(\mathrm{C}_{n}^{\omega}\right) / \sqrt{n} \stackrel{d}{\longrightarrow} \mathbf{D}\left(\mathcal{T}_{\mathrm{e}}\right)=\sup _{0 \leqslant x<y \leqslant 1}\left(\mathrm{e}_{x}+\mathrm{e}_{y}-2 \inf _{x \leqslant t \leqslant y} \mathrm{e}_{t}\right)
$$

By Aldous [4, Ch. 3.4] and Wang [38], the diameter admits the density

$$
\mathbb{P}\left(\mathbf{D}\left(\mathcal{T}_{\mathrm{e}}\right)>x\right)=\sum_{k=1}^{\infty}\left(k^{2}-1\right)\left(\frac{2}{3} k^{4} x^{4}-4 k^{2} x^{2}+2\right) \exp \left(-k^{2} x^{2} / 2\right) .
$$

Equation (5) entails $\sup _{n \geqslant 1} \mathbb{E}\left[\mathbf{D}\left(\mathrm{C}_{n}^{\omega}\right)^{p} / n^{p / 2}\right]<\infty$ for each integer $p \geqslant 1$. Hence, $\mathbf{D}\left(\mathrm{C}_{n}^{\omega}\right) / \sqrt{n}$ is $p$-uniformly integrable for all $p \geqslant 1$. Combining this fact with (7), it follows that

$$
c_{\omega}^{p} \mathbb{E}\left[\mathbf{D}\left(\mathrm{C}_{n}^{\omega}\right)^{p}\right] / n^{p / 2} \rightarrow \mathbb{E}\left[\mathbf{D}\left(\mathcal{T}_{\mathrm{e}}\right)^{p}\right]
$$


The moments of the diameter of the continuum random tree $\mathcal{T}_{\mathrm{e}}$ may be obtained by an easy calculation from results by Broutin and Flajolet [11]. They are given by

$$
\begin{aligned}
\mathbb{E}\left[\mathbf{D}\left(\mathcal{T}_{\mathrm{e}}\right)\right] & =\frac{4}{3} \sqrt{\frac{\pi}{2}}, \quad \mathbb{E}\left[\mathbf{D}\left(\mathcal{T}_{\mathrm{e}}\right)^{2}\right]=\frac{2}{3}\left(1+\frac{\pi^{2}}{3}\right), \quad \mathbb{E}\left[\mathbf{D}\left(\mathcal{T}_{\mathrm{e}}\right)^{3}\right]=2 \sqrt{2 \pi}, \\
\mathbb{E}\left[\mathbf{D}\left(\mathcal{T}_{\mathrm{e}}\right)^{p}\right] & =\frac{2^{p / 2}}{3} p(p-1)(p-3) \Gamma(p / 2)(\zeta(p-2)-\zeta(p)) \quad \text { if } p \geqslant 4 .
\end{aligned}
$$

Here $\Gamma$ denotes Euler's gamma function, and $\zeta$ denotes Riemann's zeta function.

The order $\sqrt{n}$ of the diameter of random graphs from labelled classes of subcritical graphs has been established by Drmota and Noy [16, Thm. 3.2], who also conjectured a distributional scaling limit. This conjecture was later proven by Panagiotou, S., and Weller [30]. Additive parameters of random graphs from subcritical classes of unlabelled graphs have been studied by Drmota, Fusy, Kang, Kraus, and Rué [15]. It is natural to also study their extremal parameters, and Equation (7) confirms that the conjectured behaviour of the diameter also holds in the unlabelled case.

We emphasize, however, that the scaling constant $c_{\omega}$ may change depending on whether we look at labelled or unlabelled graphs. For example, among the many classes of graphs that satisfy the tree-like Assumption (2), uniform unlabelled outerplanar graphs have received particular attention in [9]. This corresponds to the case where we assign weight 1 to each graph that may be drawn in the plane such that no edges intersect and each vertex lies on the frontier of the outer face. All other graphs receive weight 0 . We derive a numeric approximation of the scaling factor for this class of graphs.

Theorem 3. The scaling constant $c_{\omega_{\mathcal{O}}}$ of the class of unlabelled outerplanar graphs is approximately given by

$$
c_{\omega_{\mathscr{O}}} \approx 0.9864689 \text {. }
$$

This differs from the case of random labelled outerplanar graphs for which the constant is approximately given by $\approx 0.960$ [30, Prop. 8.6] and the case of random outerplanar maps for which it equals $9 /(7 \sqrt{2}) \approx 0.909[12,31]$.

Theorem 2 has further consequences: Equation (4) implies distributional joint convergence for the rescaled distances between $k$ independently sampled random points of $\mathrm{C}_{n}^{\omega}$, see [28, Prop. 10] for a justification in a more general context. For example, it follows that the graph distance $d_{\mathrm{C}_{n}^{\omega}}\left(v^{1}, v^{2}\right)$ of two independently and uniformly selected points $v^{1}, v^{2} \in \mathrm{C}_{n}^{\omega}$ satisfies

$$
c_{\omega} d_{C_{n}^{\omega}}\left(v^{1}, v^{2}\right) / \sqrt{n} \stackrel{d}{\longrightarrow} d_{\mathcal{T}_{\mathrm{e}}}\left(u^{1}, u^{2}\right),
$$

with $u^{1}, u^{2}$ denoting independent $\mu$-distributed points of the Brownian tree $\mathcal{T}_{\mathrm{e}}$. The known distribution of $d_{\mathcal{T}_{\mathrm{e}}}\left(u^{1}, u^{2}\right)$ may be derived as follows. The Brownian tree satisfies a rerooting invariance $[4$, Eq. (20)], meaning that the equivalence class $\overline{0}$ of $0 \in[0,1]$ satisfies $\left(\mathcal{T}_{\mathbf{e}}, v^{1}\right) \stackrel{d}{=}\left(\mathcal{T}_{\mathbf{e}}, \overline{0}\right)$. Hence

$$
d_{\mathcal{T}_{\mathbf{e}}}\left(u^{1}, u^{2}\right) \stackrel{d}{=} d_{\mathbf{e}}\left(0, u^{1}\right)=\mathrm{e}_{u^{1}}
$$


It follows from [18, Prop. 3.4] that standardized Brownian excursion evaluated at a uniformly and independently selected point of the unit interval follows the distribution

$$
\mathbb{P}\left(\mathrm{e}_{u^{1}} \in A\right)=\int_{A} 4 x \exp \left(-2 x^{2}\right) \mathrm{d} s
$$

for $A$ a Borel subset of $\left[0, \infty\left[\right.\right.$. Hence $2 \mathrm{e}_{u^{1}}$ follows the Rayleigh distribution with probability density $x \exp \left(-x^{2} / 2\right)$. Summing up:

$$
2 c_{\omega} d_{C_{n}^{\omega}}\left(v^{1}, v^{2}\right) / \sqrt{n} \stackrel{d}{\longrightarrow} \text { Rayleigh }(1) .
$$

Note that the tail-bound (5) implies that the distance $d_{\mathrm{C}_{n}^{\omega}}\left(v^{1}, v^{2}\right)$ is $p$-uniformly integrable for any $p \in \mathbb{N}$, yielding (similarly as we argued for the diameter) that

$$
\mathbb{E}\left[d_{C_{n}^{\omega}}\left(v^{1}, v^{2}\right)^{p}\right] \sim n^{p / 2} 2^{-p / 2} c_{\omega}^{-p} \Gamma(1+p / 2) .
$$

The Gromov-Hausdorff-Prokhorov universality class of the Brownian continuum random tree (and other continuous limit objects) was also studied in a recent work on Voronoi tesselations [2], and the results given there also apply to the random graph $\mathrm{C}_{n}^{\omega}$ by Theorem 2.

\subsection{Local limit}

Apart from a scaling limit describing the asymptotic global shape of $C_{n}^{\omega}$ we also describe the asymptotic local shape via a Benjamini-Schramm limit.

Theorem 4. Suppose that Assumption (2) holds. Then there exists a locally finite limit graph $\hat{\mathrm{C}}^{\omega}$ with a distinguished vertex $\hat{v} \in \hat{\mathrm{C}}^{\omega}$ such that $\mathrm{C}_{n}^{\omega}$ converges toward $\left(\hat{\mathrm{C}}^{\omega}, \hat{v}\right)$ in the Benjamini-Schramm sense. Even stronger, if $v_{n}$ denotes uniformly at random drawn vertex from $\mathrm{C}_{n}^{\omega}$, and $k_{n}=o(\sqrt{n})$ is a fixed deterministic sequence of non-negative integers, then

$$
\lim _{n \rightarrow \infty} d_{\mathrm{TV}}\left(U_{k_{n}}\left(\mathrm{C}_{n}^{\omega}, v_{n}\right), U_{k_{n}}\left(\hat{\mathrm{C}}^{\omega}, \hat{v}\right)\right)=0,
$$

with $U_{k_{n}}(\cdot, \cdot)$ denoting the subgraph induced by all vertices with distance at most $k_{n}$ from the specified vertex.

See Section 6.1 below for a concise introduction to local limits. The BenjaminiSchramm convergence is deduced by observing that the $k_{n}$-neighbourhood of a uniformly at random drawn vertex $v_{n} \in \mathrm{H}_{n}+\mathrm{A}_{n-h_{n}}^{\omega}$ lies with high probability entirely in $\mathrm{A}_{n-h_{n}}^{\omega}$ and hence it suffices to establish local convergence for $A_{n-h_{n}}^{\omega}$. This is achieved by using the stronger form of Benjamini-Schramm convergence for $A_{n}^{\omega}$ established in [33]. As a byproduct, we obtain that the Benjamini-Schramm limits of $A_{n}^{\omega}$ and $C_{n}^{\omega}$ agree. In particular, the distribution of the limit graph may be described via a blow-up procedure applied to a specific random infinite tree, see [33, Sec. 6.2 and Sec. 6.4.2 ].

For the case where $\iota(B) \in\{0,1\}$ for all $B$ and for which the $\omega$-weight of all trees is positive, we hence recover the local convergence of random unlabelled unrooted trees [34, Thm. 1.2] (at least in the case without vertex degree restrictions) and extend the local limit of uniform random unlabelled unrooted graphs from subcritical classes [20, Thm. 4.4 ] that states convergence of neighbourhoods with constant radius instead of $o(\sqrt{n})$. 


\subsection{Disconnected regime}

Our last result is an observation on random graphs that are not necessarily connected. That is, random unlabelled elements from the class $\mathscr{G}^{\omega}$ of $\omega$-weighted graphs, where the $\omega$ weight of such a graph is defined as the product of $\omega$-weights of its connected components. Similarly as in the approximation of unrooted graphs by rooted graphs in Theorem 1, the following limit allows us to transfer "practically every" asymptotic property from the connected to the disconnected regime.

Theorem 5. Let $\mathrm{G}_{n}^{\omega}$ denote the random unlabelled graph with $n$ vertices sampled with probability proportional to the product of $\omega$-weights of its connected components. If Assumption (2) holds, then the largest connected component $\mathrm{C}_{n}$ of $\mathrm{G}_{n}^{\omega}$ has size $\left|\mathrm{C}_{n}\right|=n+O_{p}(1)$. More precisely, let $d \geqslant 1$ denote the unique largest constant such that all finite connected graphs with positive $\omega$-weight have size in the lattice $1+d \mathbb{Z}$. Then for each $0 \leqslant a<d$ there exists a finite random graph $\mathrm{G}_{a}$ such that

$$
\mathrm{G}_{n}^{\omega}-\mathrm{C}_{n} \stackrel{d}{\longrightarrow} \mathrm{G}_{a}
$$

as $n$ tends to infinity on the lattice $a+d \mathbb{N}$. Here weak convergence is to be understood in the usual sense, that is, of random elements of the countable set of unlabelled finite graphs. The distribution of $\mathrm{G}_{a}$ has Boltzmann-type:

$$
\mathbb{P}\left(\mathrm{G}_{a}=G\right)=\omega(G) \rho_{\mathscr{A}}^{|G|}\left(\sum_{H,|H| \equiv a-1} \omega(H) \rho_{\mathscr{A}}^{|H|}\right)^{-1}, \quad|G| \equiv a-1 \bmod d \quad \bmod .
$$

Here the sum index $H$ ranges over all unlabelled graphs with size in the lattice $a-1+d \mathbb{Z}$.

Compare with a result for the number of connected components for the case of random unlabelled outerplanar graphs in [9, Thm. 5.1], and under a general smoothness condition given in [24, Chap. 4, Sec. 6.4]. It follows from Theorem 5 that the limits in Theorems 2 and 4 also hold for the largest component of $\mathrm{G}_{n}^{\omega}$, and, if we permit disconnected graphs in the notion of local weak convergence, we may also consider Theorem 4 as a limit theorem for the random graph $\mathrm{G}_{n}^{\omega}$.

A similar result was observed for random labelled graphs by McDiarmid [26] (with later generalizations in [32, Thm. 4.2]). It is natural to expect that, similarly to the labelled case, the tree-like condition (2) is not required for Theorem 5 to hold, and may be replaced by merely requiring the series $\tilde{\mathscr{A}}^{\omega}(z)$ to have positive radius of convergence. However, this appears to be an open problem.

\subsection{Notation}

Throughout we let $n$ denote a positive integer. All unspecified limits are as $n \rightarrow \infty$. We say an event (that depends on $n$ ) holds with high probability if its probability tends to 1 as $n$ becomes large. Convergence in probability and distribution (also called weak convergence) are denoted by $\stackrel{p}{\longrightarrow}$ and $\stackrel{d}{\longrightarrow}$. For any sequence $a_{n}>0$ of positive real 
numbers we let $o_{p}\left(a_{n}\right)$ denote an unspecified random variable $Z_{n}$ such that $Z_{n} / a_{n} \stackrel{p}{\longrightarrow} 0$. The total variation distance between two random variables $X$ and $Y$ taking values in a common measurable space $(M, \mathcal{F})$ is denoted by

$$
d_{\mathrm{TV}}(X, Y)=\sup _{A \in \mathcal{F}}|\mathbb{P}(X \in A)-\mathbb{P}(Y \in A)| .
$$

\section{Block-decomposition of cycle-pointed graphs}

To deal with the symmetries that complicate the analysis of unlabelled graphs, we will make use of enumerative and probabilistic aspects of the theory of species. In Appendix A we summarize some tools and notions of this theory that we require in the proofs of our main results, and provide further references for a detailed introduction to the topic.

\subsection{The block-tree}

A cut-vertex of a connected graph is a vertex whose removal disconnects the graph. We say a graph is 2-connected, if it is connected, has at least 2 vertices, but no cut-vertices. This includes the link-graph consisting of two vertices joined by an edge. A block $B$ of a graph $G$ is a subgraph that is inclusion maximal with the property of being either an isolated vertex or 2-connected. Any two blocks overlap in at most one vertex. The cutvertices of a connected graph are precisely the vertices that belong to more than one block. For any connected graph $C$ we may form the associated block-tree $T(C)$ that comes with a bipartition of its vertices into two groups of vertices [14, Ch. 3.1]. One group corresponds to the blocks of $C$ and the other to its cut-vertices. The edges of the tree $T(C)$ are given by all pairs $\{v, B\}$ with $v$ a cut-vertex and $B$ a block that contains the vertex $v$.

\subsection{Cycle pointing}

We recall the block-decomposition of cycle-pointed connected graphs by Bodirsky, Fusy, Kang and Vigerske [10, Prop. 28] and check that it is compatible with block-weightings. We assume familiarity with the cycle pointing operations, see Appendix A and the references given therein. Let $\mathscr{B}^{\iota}$ be the weighted species of graphs that are 2-connected, and let $\mathscr{C}^{\omega}$ be the weighted species of connected graphs with the $\omega$-weights given as in Equation (1).

Marking a connected graph at a 1-cycle is equivalent to marking a vertex, and hence we may split the species $\mathscr{C}^{\circ}$ of cycle-pointed connected graphs into vertex-marked graphs from the weighted class $\mathscr{A}^{\omega}$ and graphs from the species $\left(\mathscr{C}^{\circledast}\right)^{\omega}$ of cycle-pointed connected graphs whose cycle has length at least two:

$$
\left(\mathscr{C}^{\circ}\right)^{\omega} \simeq \mathscr{A}^{\omega}+\left(\mathscr{C}^{\circledast}\right)^{\omega} .
$$

Let $C$ be a connected graph that is marked at a cycle $\tau$ of length at least two. Then there exists an automorphism $\sigma$ of $C$ that has $\tau$ as one of its disjoint cycles. The automorphism $\sigma$ induces a canonical isomorphism $\bar{\sigma}$ of the properly bicolored block-tree $T(C)$ 

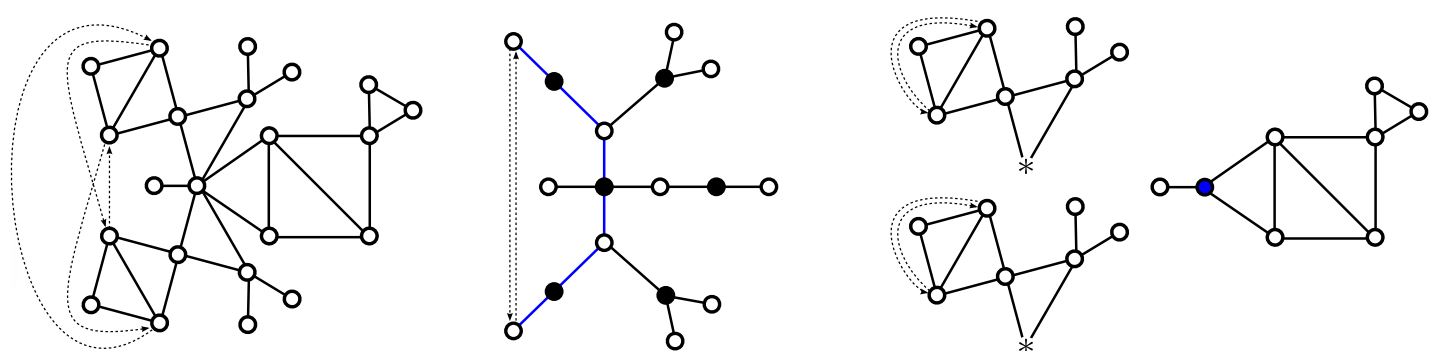

Figure 2: Illustration of Decomposition (23) for a cycle-pointed graph with a cutvertex as cycle-center.

whose white vertices correspond to the blocks, and black vertices correspond to cutvertices of $C$. To any vertex $v$ of $C$ we may assign a unique vertex $\bar{v}$ of $T(C)$, because either $v$ is a cutvertex and hence corresponds to a black vertex of $T(C)$, or $v$ is not a cutvertex, and hence is contained in a unique block of $C$ and hence corresponds to a white vertex of $T(C)$. Since $\tau$ is a cycle of $\sigma$, it follows that the vertices of $T(C)$ that correspond to the atoms of $\tau$ form a cycle $\bar{\tau}$ of the tree-automorphism $\bar{\sigma}$. The cycle $\bar{\tau}$ need not have the same length as the cycle $\tau$, as non-cutvertices of $\tau$ that lie in the same block get contracted to a single atom of $\bar{\tau}$.

For each atom $v$ of $\tau$ we may consider the unique path $P_{v}$ in the tree $T(C)$ that joins $\bar{v}$ the vertex $\tau(\bar{v})$ corresponding to the consecutive atom in the cycle. As $\bar{\sigma}$ permutes these path, they all have the same lengths. Note that either all or none of the vertices of $\tau$ are cutvertices, as the graph automorphism $\sigma$ permutes only cutvertices with cutvertices and non-cutvertices with non-cutvertices. Hence all vertices of $\bar{\tau}$ share the same colour. In a properly bicolored graph the distance between two vertices of the same colour is always an even number, hence each of the paths $P_{v}$ has an even number of edges and hence a unique center vertex. A general result given in [10, Claim 22] states that all connecting paths in a cycle-pointed tree must share the same center, so we may consider the center vertex $u$ of the connecting paths in $T(C)$. Hence the species $\mathscr{C}^{\circledast}$ may be split into two summands,

$$
\left(\mathscr{C}^{\circledast}\right)^{\omega} \simeq\left(\mathscr{C}_{v}^{\circledast}\right)^{\omega}+\left(\mathscr{C}_{b}^{\circledast}\right)^{\omega}
$$

corresponding to the subspecies where the center of the marked cycles is required to correspond to a cutvertex or to a block, respectively. Clearly the center vertex is a fixpoint of $\bar{\sigma}$, and this fact allows us to give explicit decompositions for both.

Let us first consider the case where the center $u$ corresponds to a cutvertex $v_{\tau}$. Each branch $A$ of the rooted tree $(T(C), u)$ corresponds to graph $G(A)$ with a distinguished vertex that corresponds to the vertex $v_{\tau}$ and is not a cut-vertex of $G(A)$. In order to keep the label sets disjoint, we label this vertex by a $*$-place-holder instead of $v_{\tau}$. Hence any branch is simply a derived block from $\mathscr{B}^{\prime}$ where each non-*-vertex gets identified with the root of a connected rooted graph. In other words, it's a $\mathscr{B}^{\prime} \circ \mathscr{A}$-object. Moreover, the whole graph consists simply of the center vertex $v_{\tau}$ together with an unordered symmetrically cycle-pointed collection of $\mathscr{B}^{\prime} \circ \mathscr{A}$-objects. The $\omega$-weight of $C$ is the product of the $\omega$ weights of the branches, and each automorphism of $C$ having $\tau$ as its cycle leaves the 

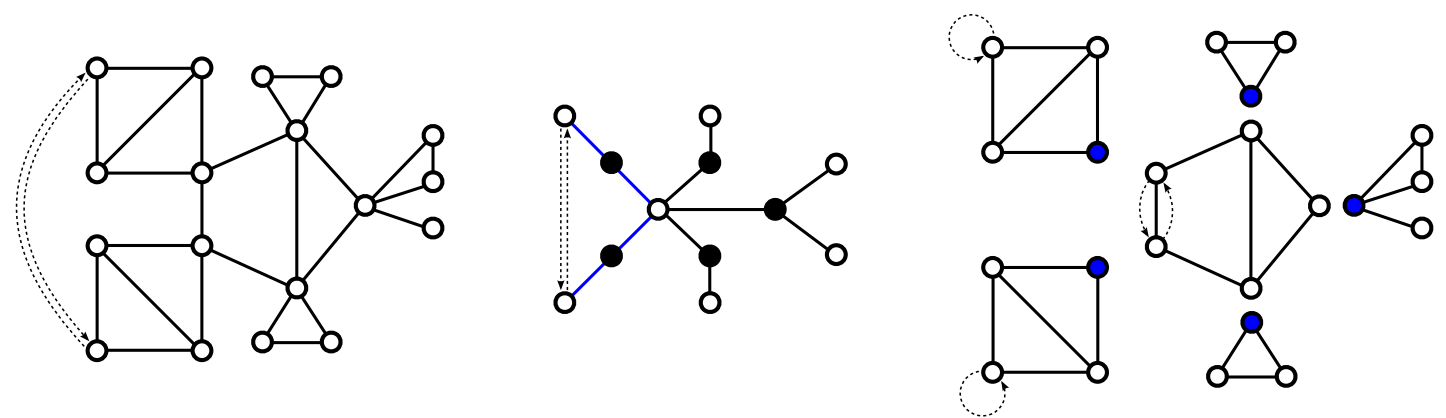

Figure 3: Illustration of Decomposition (24) for a cycle-pointed graph with a block as cycle-center.

center vertex $v_{c}$ invariant. Furthermore, the $\omega$-weight of a branch is the product of the $\iota$-weight of the derived block and the $\omega$-weight of the attached rooted connected graphs. Hence

$$
\left(\mathscr{C}_{v}^{\circledast}\right)^{\omega} \simeq\left(\mathrm{SET}^{\circledast} \odot\left(\left(\mathscr{B}^{\prime}\right)^{\iota} \circ \mathscr{A}^{\omega}\right)\right) \star \mathscr{X}
$$

where the factor $\mathscr{X}$ corresponds to the center vertex. We may write

$$
\mathrm{SET}^{\circledast} \simeq \mathscr{P} \star \mathrm{SET}
$$

with $\mathscr{P}$ denoting the cycle-pointed species consisting only of marked cycles with length at least two, which simplifies (21) to

$$
\left(\mathscr{C}_{v}^{\circledast}\right)^{\omega} \simeq\left(\mathscr{P} \odot\left(\left(\mathscr{B}^{\prime}\right)^{\iota} \circ \mathscr{A}^{\omega}\right)\right) \star \mathscr{A}^{\omega} .
$$

This corresponds to the fact that the center together with the branches without any atoms of $\tau$ form a connected rooted graph without any further restrictions, and the remaining branches together with the marked cycle $\tau$ correspond to a $\mathscr{P} \odot \mathscr{B}^{\prime} \circ \mathscr{A}$ object. Furthermore, this object may be composed out of a single cycle-pointed $\left(\mathscr{B}^{\prime} \circ \mathscr{A}\right)^{\circ}$ object by constructing $\tau$ according to the cycle composition construction, see Figure 2 for an illustration.

Finally, consider the case where the center $u$ corresponds to a block $B$ instead of a cutvertex. There is a natural marked cycle $\tau_{B}$ on the block $B$. It is given by the cycle $\tau$ if $\tau$ lies entirely in $B$. Otherwise, $\tau_{B}$ consists of the cutvertices of $C$ that are contained in $B$ and belong to those branches in $T(C)$ adjacent to the center $u$, that contain atoms of the induced cycle $\bar{\tau}$. This is because the induced automorphism $\bar{\sigma}$ of $T(C)$ permutes the branches containing atoms of $\bar{\tau}$ cyclically. The graph automorphism $\sigma$ maps the vertex set of $B$ to itself, and hence induces an automorphism $\left.\sigma\right|_{B}$ of the block $B$. The cycle $\tau_{B}$ is one of the disjoint cycles of $\sigma_{B}$. Hence $\left(B, \tau_{B}\right)$ is a cycle-pointed block. The graph $C$ may be decomposed into the cycle-pointed block $\left(B, \tau_{B}\right)$, where each vertex $v$ of $B$ is identified with the root of a connected rooted graph $C_{v}$. The marked cycle $\tau_{B}$ and the rooted graphs corresponding to it are composed out of a single cycle-pointed rooted connected graph $C_{\tau}$ according to the cycle composition construction (see Figure 3 below 
for an illustration). Furthermore, the $\omega$-weight of the graph $C$ is given by the product of the $\iota$-weight of $B$ and the $\omega$-weights of the attached graphs $\left(C_{v}\right)_{v \in B}$. Summing up, we obtain the decomposition

$$
\left(\mathscr{C}_{b}^{\circledast}\right)^{\omega} \simeq\left(\mathscr{B}^{\circledast}\right)^{\iota} \odot \mathscr{A}^{\omega} .
$$

\subsection{The case of a vertex cycle-center}

Throughout this section, we assume that Assumption (2) is satisfied. Equation (23) states that an unlabelled symmetrically cycle-pointed weighted connected graph may be decomposed uniquely in a weight-preserving manner into an unlabelled rooted graph and an unlabelled graph from the species $\mathscr{P} \odot\left(\left(\mathscr{B}^{\prime}\right)^{\iota} \circ \mathscr{A}^{\omega}\right)$. We are going to verify that the sum of the weights of $n$-sized graphs from this species is exponentially smaller than the sum of the $\omega$-weights of $n$-sized unlabelled rooted graphs. This may then be used to show that large random unlabelled graphs from $\left(\mathscr{C}_{v}^{\circledast}\right)^{\omega}$ consist of a large rooted graph together with a stochastically bounded rest attached to its root.

Recall that $d$ denotes the span of the support of the generating series $\tilde{\mathscr{C}}^{\omega}(z)$. That is, $d \geqslant 1$ is minimal with the property that the exponents with non-zero coefficients belong to the lattice $1+d \mathbb{Z}$. By a standard result due to Bell, Burris and Yeats [6, Thm. 28] we know that the tree-like Assumption (2) implies that there exists a constant $c_{\mathscr{A}}>0$ such that

$$
\left[z^{n}\right] \tilde{\mathscr{A}}^{\omega}(z) \sim c_{\mathscr{A}} n^{-3 / 2} \rho_{\mathscr{A}}^{-n}
$$

as $n \equiv 1 \bmod d$ becomes large.

Lemma 6. The ordinary generating series

$$
\widehat{\mathscr{P} \odot\left(\left(\mathscr{B}^{\prime}\right)^{l} \circ \mathscr{A}^{\omega}\right)(z)}=\sum_{i=2}^{\infty} \bar{Z}_{\left(\left(\mathcal{B}^{\prime}\right)^{\circ}\right)^{\iota}}\left(\tilde{\mathscr{A}}^{\omega^{i}}\left(z^{i}\right),\left(\tilde{\mathscr{A}}^{\circ}\right)^{\omega^{i}}\left(z^{i}\right) ; \tilde{\mathscr{A}}^{2 i}\left(z^{2 i}\right),\left(\tilde{\mathscr{A}}^{\circ}\right)^{\omega}\left(z^{2 i}\right) ; \ldots\right)
$$

has radius of convergence strictly larger than $\rho_{\mathscr{A}}$.

Here the wide tilde indicates that we look at the ordinary generating series of the entire species $\mathscr{P} \odot\left(\left(\mathscr{B}^{\prime}\right)^{\iota} \circ \mathscr{A}^{\omega}\right)$.

Proof. For any $k \geqslant 1$ and any rooted symmetry $(B, \sigma, \tau, v) \in \operatorname{RSym}\left(\left(\left(\mathscr{B}^{\prime}\right)^{\circ}\right)^{\iota}\right)[k]$ it holds that

$$
\begin{aligned}
{\left[z^{b}\right]\left(\tilde{\mathscr{A}}^{\circ}\right)^{\omega^{|\tau|}}\left(z^{|\tau|}\right)\left(\tilde{\mathscr{A}}^{\omega}(z)\right)^{\sigma_{1}} \cdots\left(\tilde{\mathscr{A}}^{\omega^{|\tau|}}\left(z^{|\tau|}\right)\right)^{\sigma_{|\tau|}-1} \cdots } & \left(\tilde{\mathscr{A}}^{\omega^{k}}\left(z^{k}\right)\right)^{\sigma_{k}} \\
& \leqslant b\left[z^{b}\right]\left(\tilde{\mathscr{A}}^{\omega}(z)\right)^{\sigma_{1}} \cdots\left(\tilde{\mathscr{A}}^{\omega^{k}}\left(z^{k}\right)\right)^{\sigma_{k}} .
\end{aligned}
$$

Here we have applied the fact that each unlabelled $\mathscr{A}$-structure of size $\ell \geqslant 1$ has precisely $\ell$ cycle pointings. To any symmetry of size $k$ correspond precisely $k$ rooted symmetries. 
It follows that for any $b \geqslant 1$

$$
\begin{aligned}
& \quad\left[z^{b}\right] \sum_{\substack{k \geqslant 1 \\
(B, \sigma, \tau, v) \in \operatorname{RSym}\left(\left(\left(\mathscr{B}^{\prime}\right)^{\circ}\right)^{\iota}\right)[k]}} \frac{1}{k !} \iota(B)\left(\tilde{\mathscr{A}}^{\circ}\right)^{\omega^{|\tau|}}\left(z^{|\tau|}\right)\left(\tilde{\mathscr{A}}^{\omega}(z)\right)^{\sigma_{1}} \cdots\left(\tilde{\mathscr{A}}^{|\tau|}\left(z^{|\tau|}\right)\right)^{\sigma|\tau|}-1 \cdots\left(\tilde{\mathscr{A}}^{\omega^{k}}\left(z^{k}\right)\right)^{\sigma_{k}} \\
& \leqslant\left[z^{b}\right] \sum_{k \geqslant 1} \frac{b k}{k !} \sum_{(B, \sigma) \in \operatorname{Sym}\left(\left(\mathscr{B}^{\prime}\right)^{\iota}\right)[k]}\left(\tilde{\mathscr{A}}^{\omega}(z)\right)^{\sigma_{1}} \cdots\left(\tilde{\mathscr{A}}^{\omega^{k}}\left(z^{k}\right)\right)^{\sigma_{k}}
\end{aligned}
$$

Only summands with $\left[z^{b}\right]\left(\tilde{\mathscr{A}}^{\omega}(z)\right)^{\sigma_{1}} \ldots \tilde{\mathscr{A}}^{\omega^{k}}\left(z^{k}\right)^{\sigma_{k}} \neq 0$ contribute. As $\sigma_{1}+2 \sigma_{2}+\ldots+k \sigma_{k}=$ $k$, this means that we only need to consider summands where $k \leqslant b$. Thus

$$
\begin{aligned}
{\left[z^{b}\right] \bar{Z}_{\left(\left(\mathcal{B}^{\prime}\right)^{\circ}\right)^{\iota}}\left(\tilde{\mathscr{A}}^{\omega}(z),\left(\tilde{\mathscr{A}}^{\circ}\right)^{\omega}(z) ; \tilde{\mathscr{A}}^{\omega}\left(z^{2}\right),\left(\tilde{\mathscr{A}}^{\circ}\right)^{\omega}\left(z^{2}\right)\right.} & ; \ldots) \\
& \leqslant b^{2}\left[z^{b}\right] Z_{\left(\mathcal{B}^{\prime}\right)^{\iota}}\left(\tilde{\mathscr{A}}^{\omega}(z), \tilde{\mathscr{A}}^{\omega}\left(z^{2}\right), \ldots\right) .
\end{aligned}
$$

It follows that for any $\epsilon>0$

$$
\begin{aligned}
\overline{\mathscr{P} \odot\left(\left(\mathscr{B}^{\prime}\right)^{\iota} \circ(\mathscr{A})^{\omega}\right)} & \left(\rho_{\mathscr{A}}+\frac{\epsilon}{2}\right) \\
& \leqslant \sum_{i \geqslant 2} \sum_{b \geqslant 1} b^{2}\left(\rho_{\mathscr{A}}+\frac{\epsilon}{2}\right)^{b i}\left[z^{b i}\right] Z_{\left(\mathcal{B}^{\prime}\right)^{\iota}}\left(\tilde{\mathscr{A}}^{\omega}\left(z^{i}\right), \tilde{\mathscr{A}}^{\omega}\left(z^{2 i}\right), \ldots\right) \\
& =\sum_{i \geqslant 2} \frac{1}{i} \sum_{b \geqslant 1} b^{2} i\left(\frac{\rho_{\mathscr{A}}+\frac{\epsilon}{2}}{\rho_{\mathscr{A}}+\epsilon}\right)^{b i}\left(\rho_{\mathscr{A}}+\epsilon\right)^{b i}\left[z^{b i}\right] Z_{\left(\mathcal{B}^{\prime}\right)^{\iota}}\left(\tilde{\mathscr{A}}^{\omega}\left(z^{i}\right), \tilde{\mathscr{A}}^{\omega}\left(z^{2 i}\right), \ldots\right) .
\end{aligned}
$$

Clearly it holds that $b i\left(\frac{\rho_{\mathscr{A}}+\frac{\epsilon}{2}}{\rho_{\mathscr{A}}+\epsilon}\right)^{b i}<1$ for all but finitely many pairs $(i, b)$. It follows by Assumption (2) that the upper bound in (27) is finite for $\epsilon$ small enough.

The asymptotic expansion (25) and Lemma 6 allow us to apply a standard result [19, Thm. VI.12] on the coefficients of products of power series, yielding

$$
\left[z^{n}\right]\left(\tilde{\mathscr{C}}_{v}^{\circledast}\right)^{\omega}(z) \sim \overline{\mathscr{P} \odot\left(\left(\mathscr{B}^{\prime}\right)^{l} \circ \mathscr{A}^{\omega}\right)}\left(\rho_{\mathscr{A}}\right)\left[z^{n}\right] \mathscr{A}^{\omega}(z)
$$

as $n$ becomes large. We may apply this to show that large random $\left(\mathscr{C}_{v}^{\circledast}\right)^{\omega}$-objects look like large $\mathscr{A}^{\omega}$-objects with a stochastically bounded rest attached to the root.

Lemma 7. The $\mathscr{P} \odot\left(\left(\mathscr{B}^{\prime}\right)^{\iota} \circ \mathscr{A}^{\omega}\right)$-object corresponding to a random unlabelled $n$-sized $\left(\mathscr{C}_{v}^{\circledast}\right)^{\omega}$-object that is sampled with probability proportional to its weight has stochastically bounded size.

Proof. By the asymptotic expansions (25) and (28) it follows that the probability for this component to have size $k$ is asymptotically given by

$$
\frac{\left(\left[z^{n-k}\right] \tilde{\mathscr{A}}^{\omega}(z)\right)\left(\left[z^{k}\right] \overline{\mathscr{P} \odot\left(\left(\mathscr{B}^{\prime}\right)^{\iota} \circ \mathscr{A}^{\omega}\right)}(z)\right)}{\left[z^{n}\right] \tilde{\mathscr{C}}_{v}^{\circledast}(z)} \rightarrow \frac{\rho_{\mathscr{A}}^{k}\left[z^{k}\right] \overline{\mathscr{P} \odot\left(\left(\mathscr{B}^{\prime}\right)^{\iota} \circ \mathscr{A}^{\omega}\right)}(z)}{\overline{\mathscr{P} \odot\left(\left(\mathscr{B}^{\prime}\right)^{\iota} \circ \mathscr{A}^{\omega}\right)}\left(\rho_{\mathscr{A}}\right)} .
$$


As the limit probabilities sum to 1, this implies that the component size has a finite weak limit and is hence stochastically bounded. (In fact, this even shows that the component converges weakly to a limit graph following a Boltzmann distribution for unlabelled $\mathscr{P} \odot$ $\left(\left(\mathscr{B}^{\prime}\right)^{\iota} \circ \mathscr{A}^{\omega}\right)$-objects with parameter $\left.\rho_{\mathscr{A}} \cdot\right)$

\subsection{The case of a block cycle-center}

Throughout we assume that Assumption (2) is satisfied. We start with the following subcriticality observation.

Lemma 8. The bivariate power series

$$
f(x, y):=\bar{Z}_{(\mathscr{B} \circledast)^{\iota}}\left(x, 0 ; \tilde{\mathscr{A}}^{\omega^{2}}\left(y^{2}\right),\left(\tilde{\mathscr{A}}^{\circ}\right)^{\omega^{2}}\left(y^{2}\right) ; \tilde{\mathscr{A}}^{3}\left(y^{3}\right),\left(\tilde{\mathscr{A}}^{\circ}\right)^{\omega^{3}}\left(y^{3}\right) ; \ldots\right)
$$

satisfies $f\left(\tilde{\mathscr{A}}^{\omega}\left(\rho_{\mathscr{A}}\right)+\epsilon, \rho_{\mathscr{A}}+\epsilon\right)<\infty$ for some $\epsilon>0$.

Proof. Any unlabelled $\mathscr{A}$-structure of size $\ell \geqslant 1$ has precisely $\ell$ cycle pointings. Hence for any $k \geqslant 1$ and any rooted symmetry $(B, \sigma, \tau, v) \in \operatorname{RSym}\left(\mathscr{B}^{\circledast}\right)[k]$ it holds that

$$
\begin{aligned}
{\left[x^{a} y^{b}\right] x^{\sigma_{1}}\left(\tilde{\mathscr{A}}^{\circ}\right)^{\omega^{|\tau|}}\left(y^{|\tau|}\right)\left(\tilde{\mathscr{A}}^{\omega^{2}}\left(y^{2}\right)\right)^{\sigma_{2}} \cdots\left(\tilde{\mathscr{A}}^{|\tau|}\left(y^{|\tau|}\right)\right)^{\sigma_{|\tau|}-1} \cdots \tilde{\mathscr{A}}^{\omega^{k}}\left(y^{k}\right)^{\sigma_{k}} } & \\
& \leqslant b\left[x^{a} y^{b}\right] x^{\sigma_{1}}\left(\tilde{\mathscr{A}}^{\omega^{2}}\left(y^{2}\right)\right)^{\sigma_{2}} \cdots \tilde{\mathscr{A}}^{\omega^{k}}\left(y^{k}\right)^{\sigma_{k}}
\end{aligned}
$$

Any symmetry from $\operatorname{Sym}(\mathscr{B})[k]$ corresponds to precisely $k$ rooted symmetries, so any nontrivial symmetry may correspond to at most $k$ rooted symmetry from the symmetrically cycle-pointed species $\left(\mathscr{B}^{\circledast}\right)^{\iota}$. Hence

$$
\begin{aligned}
& {\left[x^{a} y^{b}\right] f(x, y)} \\
& =\left[x^{a} y^{b}\right] \sum_{\substack{k \geqslant 2 \\
(B, \sigma, \tau, v) \in \operatorname{RSym}(\mathscr{B} \circledast)[k]}} \frac{\iota(B)}{k !} x^{\sigma_{1}}\left(\tilde{\mathscr{A}}^{\circ}\right)^{\omega^{|\tau|}}\left(y^{|\tau|}\right)\left(\tilde{\mathscr{A}}^{\omega^{2}}\left(y^{2}\right)\right)^{\sigma_{2}} \cdots\left(\tilde{\mathscr{A}}^{\omega^{|\tau|}}\left(y^{|\tau|}\right)\right)^{\sigma_{|\tau|}-1} \cdots \tilde{\mathscr{A}}^{\omega^{k}}\left(y^{k}\right)^{\sigma_{k}} \\
& \leqslant\left[x^{a} y^{b}\right] \sum_{k \geqslant 2} \frac{b k}{k !} \sum_{(B, \sigma) \in \operatorname{Sym}(\mathscr{B})[k]} \iota(B) x^{\sigma_{1}}\left(\tilde{\mathscr{A}}^{\omega^{2}}\left(y^{2}\right)\right)^{\sigma_{2}} \cdots \tilde{\mathscr{A}}^{\omega^{k}}\left(y^{k}\right)^{\sigma_{k}}
\end{aligned}
$$

We may neglect any summands where $\sigma_{1} \neq a$ or $\left[y^{b}\right]\left(\tilde{\mathscr{A}} \omega^{2}\left(y^{2}\right)\right)^{\sigma_{2}} \cdots \tilde{\mathscr{A}}^{\omega^{k}}\left(y^{k}\right)^{\sigma_{k}}=0$. Since it holds that $\sigma_{1}+2 \sigma_{2}+\ldots+k \sigma_{k}=k$, this means that we only need to consider summands where $k \leqslant a+b$. Thus

$$
\left[x^{a} y^{b}\right] f(x, y) \leqslant b(a+b)\left[x^{a} y^{b}\right] Z_{\mathscr{B}^{c}}\left(x, \tilde{\mathscr{A}}^{\omega^{2}}\left(y^{2}\right), \tilde{\mathscr{A}}^{\omega^{3}}\left(y^{3}\right), \ldots\right) .
$$

It follows from the identity $Z_{\left(\mathscr{B}^{\prime}\right)^{\iota}}\left(s_{1}, s_{2}, \ldots\right)=\frac{\partial}{\partial s_{1}} Z_{\mathscr{B}^{\iota}}\left(s_{1}, s_{2}, \ldots\right)$ that for $a>0$

$$
\left[x^{a} y^{b}\right] f(x, y) \leqslant \frac{b(a+b)}{a}\left[x^{a-1} y^{b}\right] Z_{\left(\mathscr{B}^{\prime}\right)^{\iota}}\left(x, \tilde{\mathscr{A}}^{\omega^{2}}\left(y^{2}\right), \tilde{\mathscr{A}}^{\omega^{3}}\left(y^{3}\right), \ldots\right) .
$$

In order to treat the case $a=0$, we observe that the series $Z_{\mathscr{B}^{\iota}}\left(0, \tilde{\mathscr{A}}^{\omega^{2}}\left(y^{2}\right), \tilde{\mathscr{A}}^{\omega^{3}}\left(y^{3}\right), \ldots\right)$ is the sum of weight-monomials of all fixed-point-free symmetries of block-rooted connected 
graphs. We may convince ourselves of this fact as follows. Block-rooted connected graphs consist of a block with rooted graphs attached to it, so they correspond to the composition species $\mathscr{B}^{\iota} \circ \mathscr{A}^{\omega}$. By the composition formula the cycle index sum of this species is given by

$$
Z_{\mathscr{B}^{\iota}}\left(Z_{\mathscr{A} \omega}\left(s_{1}, s_{2}, \ldots\right), Z_{\mathscr{A} \omega^{2}}\left(s_{2}, s_{4}, \ldots\right), Z_{\mathscr{A} \omega^{3}}\left(s_{3}, s_{6}, \ldots\right) \ldots\right) .
$$

If we want to sum only the weight-monomials of fixed-point-free symmetries, we have to make the substitution $s_{1}=0$. But $Z_{\mathscr{A} \omega}\left(0, s_{2}, \ldots\right)=0$ as any automorphism of a rooted graph from $\mathscr{A}^{\omega}$ is required to fix the root. So $Z_{\mathscr{B}^{c}}\left(0, Z_{\mathscr{A} \omega^{2}}\left(s_{2}, s_{4}, \ldots\right), Z_{\mathscr{A} \omega^{3}}\left(s_{3}, s_{6}, \ldots\right) \ldots\right)$. is the sum of weight-monomials of fixed-point-free symmetries of block-rooted graphs. If we want to index according to the number of vertices we have to make the substitution $s_{i}=y^{i}$ for all $i \geqslant 2$, yielding $Z_{\mathscr{B}}\left(0, \tilde{\mathscr{A}}^{\omega^{2}}\left(y^{2}\right), \tilde{\mathscr{A}} \omega^{3}\left(y^{3}\right), \ldots\right)$.

Now, any connected graph with $b$ vertices has at most $b$ blocks. Hence this series counts each fixed-point-free symmetry of a connected graph with $b$ vertices (without a block-root) at most $b$ times, yielding

$$
\left[y^{b}\right] Z_{\mathscr{B}^{\iota}}\left(0, \tilde{\mathscr{A}}^{\omega^{2}}\left(y^{2}\right), \tilde{\mathscr{A}}^{\omega^{3}}\left(y^{3}\right), \ldots\right) \leqslant b\left[y^{b}\right] Z_{\mathscr{C} \omega}\left(0, y^{2}, y^{3}, \ldots\right)
$$

By (29) we may deduce

$$
\left[y^{b}\right] f(0, y) \leqslant b^{3} Z_{\mathscr{C} \omega}\left(0, y^{2}, y^{3}, \ldots\right)
$$

It follows from the bounds (30), (31) and the tree-like Assumption (2) that $f\left(\tilde{\mathscr{A}}^{\omega}\left(\rho_{\mathscr{A}}\right)+\right.$ $\left.\epsilon, \rho_{\mathscr{A}}+\epsilon\right)<\infty$ for some $\epsilon>0$.

Note that it may happen that $\bar{Z}_{\left(\left(\mathscr{B}^{\prime}\right)^{\circledast}\right)^{\iota}}=0$. This is the case if we only assign positive $\iota$-weights to graphs with the property, that any automorphism with a fixed-point must be the trivial automorphism. There are even graphs like the Frucht graph who only admit the trivial automorphism, hence we have to be mindful of this possibility.

Lemma 9. If $\bar{Z}_{\left(\left(\mathscr{B}^{\prime}\right) \circledast\right)^{\iota}}=0$, then the generating series $\left(\tilde{\mathscr{C}}_{b}^{\circledast}\right)^{\omega}(z)$ is analytic at $\rho_{\mathscr{A}}$.

Proof. The assumption $\bar{Z}_{\left(\left(\mathscr{B}^{\prime}\right)^{\circledast}\right)^{\iota}}=0$ implies that

$$
\bar{Z}_{(\mathscr{B} \circledast)^{\iota}}\left(x_{1}, y_{1} ; x_{2}, y_{2} ; \ldots\right)=\bar{Z}_{(\mathscr{B} \circledast)^{\iota}}\left(0,0 ; x_{2}, y_{2} ; \ldots\right) .
$$

Hence $\left(\tilde{\mathscr{C}}_{b}^{\circledast}\right)^{\omega}(z)=f(0, z)$. By Inequality (31) and the tree-like Assumption (2) we know that $f(0, z)$ has radius of convergence strictly larger than $\rho_{\mathscr{A}}$, and consequently so does $\left(\tilde{\mathscr{C}}_{b}^{\circledast}\right)^{\omega}(z)$.

Let us assume for the remaining part of this subsection that $\bar{Z}_{\left(\left(\mathscr{B}^{\prime}\right)^{\circledast}\right)^{\iota}} \neq 0$. In $[36$, Thm. 3.1, Lem. 3.2] general results for the behaviour of component sizes and partitions functions of unlabelled composite structures were given. Lemma 8 gives an analogous subcriticality condition to this setting, but for the cycle-pointed composition $\left(\mathscr{B}^{\circledast}\right)^{\iota} \odot \mathscr{A}^{\omega}$ rather than a regular composition. However, the arguments used in [36] may be modified to encompass the present setting. In the following we describe these modifications. 
Decomposition (24) allows us to apply the substitution rule for Boltzmann samplers given in [10, Fig. 13] in order to devise a sampling procedure for graphs from the class $\left(\mathscr{C}_{b}^{\circledast}\right)^{\omega}$. (To be precise, the results of [10] were stated in a setting of species without weights. However, the generalization to weighted species is straight-forward. See also [8].) This yields the following procedure which samples a random unlabelled $\left(\mathscr{C}_{b}^{\circledast}\right)^{\omega}$-object $\mathrm{C}$ with distribution given by

$$
\mathbb{P}(\mathrm{C}=C)=\omega(C) \rho_{\mathscr{A}}^{|C|} / \tilde{\mathscr{C}}_{b}^{\circledast}\left(\rho_{\mathscr{A}}\right) .
$$

1. Draw a rooted symmetry $(B, \sigma, \tau, v) \in \bigcup_{k \geqslant 0} \operatorname{RSym}\left(\mathscr{B}^{\circledast}\right)[k]$ with probability proportional to the weight

$$
\frac{\iota(B)}{|B| !}\left(\tilde{\mathscr{A}}^{\circ}\right)^{\omega^{|\tau|}}\left(\rho_{\mathscr{A}}^{|\tau|}\right)\left(\tilde{\mathscr{A}}^{\omega}\left(\rho_{\mathscr{A}}\right)\right)^{\sigma_{1}}\left(\tilde{\mathscr{A}}^{\omega^{2}}\left(\rho_{\mathscr{A}}^{2}\right)\right)^{\sigma_{2}} \cdots\left(\tilde{\mathscr{A}}^{\omega^{|\tau|}}\left(\rho_{\mathscr{A}}^{|\tau|}\right)\right)^{\sigma_{|\tau|}-1} \cdots \tilde{\mathscr{A}}^{\omega^{|B|}}\left(\rho_{\mathscr{A}}^{|B|}\right)^{\sigma_{|B|}} .
$$

2. For each unmarked cycle $c \neq \tau$ of $\sigma$, draw an unlabelled $\mathscr{A}^{\omega}$-object $A_{c}$ with probability proportional to the weight $\omega\left(A_{c}\right)^{|c|} \rho_{\mathscr{A}}^{|c|\left|A_{c}\right|}$. Draw a cycle-pointed graph $\left(A_{\tau}, c_{\tau}\right)$ from the unlabelled $\left(\mathscr{A}^{\circ}\right)^{\omega}$-objects with probability proportional to the weight $\omega\left(A_{\tau}\right)^{|\tau|} \rho_{\mathscr{A}}^{|\tau|\left|A_{\tau}\right|}$.

3. Construct the final graph $\mathrm{C}$ by identifying for each cycle $c$ of $\sigma$ and each atom $u \in c$ (which is a vertex of $B$ ) the vertex $u$ with the root of a copy of $A_{c}$. The marked cycle of $C$ has length $|\tau|\left|c_{\tau}\right|$ and is constructed in a certain way out of the atoms of the $|\tau|$ copies of the cycle $c_{\tau}$. (The precise way of composing this cycle is irrelevant for our following arguments. Hence we refer the reader to [10, Fig. 13] for details.)

We may split the third step into two steps 3' and 3", where in step 3' we treat only cycles $c$ of $\sigma$ of length at least two, and in step 3" we attach only the graphs $A_{c}$ for $c$ a fixed-point of $\sigma$. This way, we end up with a graph $\mathrm{H}$ in step 3' having a number $F$ of marked vertices, each of which gets identified in step 3" with the root of an independent copy of a random unlabelled $\mathscr{A}$-object $\mathrm{A}$ with distribution

$$
\mathbb{P}(\mathrm{A}=A)=\omega(A) \rho_{\mathscr{A}}^{|A|} / \tilde{\mathscr{A}}^{\omega}\left(\rho_{\mathscr{A}}\right) .
$$

The joint probability generating series for $F$ and $H:=|\mathrm{H}|-F$ is given by

$$
\mathbb{E}\left[x^{F} y^{H}\right]=f\left(x \tilde{\mathscr{A}}^{\omega}\left(\rho_{\mathscr{A}}\right), y \rho_{\mathscr{A}}\right) /\left(\tilde{\mathscr{C}}_{b}^{\circledast}\right)^{\omega}\left(\rho_{\mathscr{A}}\right) .
$$

(Recall that the bivariate power series $f$ was defined in Lemma 8.) Lemma 8 ensures that the vector $(F, H)$ has finite exponential moments. Let $\left(\mathrm{A}_{i}\right)_{i \geqslant 1}$ denote independent copies of A. Then

$$
|\mathrm{C}| \stackrel{d}{=} H+\sum_{i=1}^{F}\left|\mathrm{~A}_{i}\right| .
$$


We are now in the same situation as in [36, Eq. (4.2)], yielding by analogous arguments as for [36, Eq. (4.4)] that

$$
\mathbb{P}(|\mathrm{C}|=n) \sim \mathbb{E}[F] \mathbb{P}(|\mathrm{A}|=n)
$$

as $n \equiv 1 \bmod d$ tends to infinity. Using the identity

$$
\bar{Z}_{\left(\left(\mathscr{B}^{\prime}\right)^{\circledast}\right)^{\iota}}\left(x_{1}, y_{1} ; x_{2}, y_{2} ; \ldots\right)=\frac{\partial}{\partial x_{1}} \bar{Z}_{(\mathscr{B} \circledast)^{\iota}}\left(x_{1}, y_{1} ; x_{2}, y_{2} ; \ldots\right)
$$

this may be expressed in terms of coefficients of generating series by

$$
\frac{\left[z^{n}\right]\left(\tilde{\mathscr{C}}_{b}^{\circledast}\right)^{\omega}(z)}{\left[z^{n}\right] \tilde{\mathscr{A}}^{\omega}(z)} \rightarrow \widehat{\left(\left(\mathscr{B}^{\prime}\right)^{\circledast}\right)^{l} \odot \mathscr{A}^{\omega}}\left(\rho_{\mathscr{A}}\right) .
$$

This also holds in the case $\bar{Z}_{\left(\left(\mathscr{B}^{\prime}\right)^{\circledast}\right)^{\iota}} \neq 0$, since then $\overline{\left(\left(\mathscr{B}^{\prime}\right)^{\circledast}\right)^{\iota} \odot \mathscr{A}^{\omega}}\left(\rho_{\mathscr{A}}\right)=0$. As we are in the setting (34), we may also argue entirely analogously as in the proof of [36, Thm. 3.1] to obtain the following result.

Lemma 10. If $\bar{Z}_{\left(\left(\mathscr{B}^{\prime}\right)^{\circledast \iota}\right.} \neq 0$, then $\left(\max \left(\left|\mathrm{A}_{1}\right|, \ldots,\left|\mathrm{A}_{F}\right|\right)|| \mathrm{C} \mid=n\right)=n+O_{p}(1)$.

That is, if we draw a graph $\mathrm{C}_{n}^{b}$ with probability proportional to its weight among all unlabelled $n$-vertex graphs from the class $\left(\tilde{\mathscr{C}}_{b}^{\circledast}\right)^{\omega}$, then $\mathrm{C}_{n}^{b} \stackrel{d}{=}(\mathrm{C}|| \mathrm{C} \mid=n)$ consists of large rooted graph $\mathrm{A}_{n}^{b}$ (the component $\mathrm{A}_{i}$ with maximal size) with a small graph of stochastically bounded size attached to its root. Furthermore, for any $k \geqslant 1$ it holds that the conditioned rooted component $\left(\mathrm{A}_{n}^{b}|| \mathrm{A}_{n}^{b} \mid=k\right)$ gets sampled with probability proportional to its $\omega$-weight among all unlabelled rooted graphs with size $k$.

\section{A giant connected component}

In this section we provide a proof of Theorem 5 .

Throughout we assume that Assumption (2) is satisfied. It follows from Decompositions (19) and (20), and Equation (28), Lemma 9 and Equation (36) that

$$
\begin{aligned}
{\left[z^{n}\right] \tilde{\mathscr{C}}^{\omega}(z) } & =n^{-1}\left[z^{n}\right]\left(\tilde{\mathscr{A}^{\omega}}(z)+\left(\tilde{\mathscr{C}}_{v}^{\circledast}\right)^{\omega}(z)+\left(\tilde{\mathscr{C}}_{b}^{\circledast}\right)^{\omega}(z)\right) \\
& \sim\left(1+\widehat{\mathscr{P} \odot\left(\left(\mathscr{B}^{\prime}\right)^{l} \circ \mathscr{A}^{\omega}\right)}\left(\rho_{\mathscr{A}}\right)+\widehat{\left(\left(\mathscr{B}^{\prime}\right)^{\circledast}\right)^{l} \odot \mathscr{A}^{\omega}}\left(\rho_{\mathscr{A}}\right)\right) n^{-1}\left[z^{n}\right] \tilde{\mathscr{A}}^{\omega}(z) \\
& \sim c_{\mathscr{A}}\left(1+\widehat{\mathscr{P} \odot\left(\left(\mathscr{B}^{\prime}\right)^{\iota} \circ \mathscr{A}^{\omega}\right)}\left(\rho_{\mathscr{A}}\right)+\widehat{\left(\left(\mathscr{B}^{\prime}\right)^{\circledast}\right)^{l} \odot \mathscr{A}^{\omega}}\left(\rho_{\mathscr{A}}\right)\right) n^{-5 / 2} \rho_{\mathscr{A}}^{-n}
\end{aligned}
$$

as $n \equiv 1 \bmod d$ becomes large. The result [6, Thm. 28] by Bell, Burris and Yeats yields an explicit expression for the constant $c_{\mathscr{A}}$ in Equation (25), namely

$$
c_{\mathscr{A}}=d \sqrt{\frac{\rho_{\mathscr{A}} E_{z}\left(\rho_{\mathscr{A}}, \tilde{\mathscr{A}}^{\omega}\left(\rho_{\mathscr{A}}\right)\right)}{2 \pi E_{u u}\left(\rho_{\mathscr{A}}, \tilde{\mathscr{A}}^{\omega}\left(\rho_{\mathscr{A}}\right)\right)}}
$$


with $E_{z}$ and $E_{u u}$ denoting partial derivatives of the bivariate power series

$$
E(z, u):=z \exp \left(Z_{\left(\mathcal{B}^{\prime}\right)^{\iota}}\left(u, \tilde{\mathscr{A}}^{\omega^{2}}\left(z^{2}\right), \tilde{\mathscr{A}}^{\omega^{3}}\left(z^{3}\right), \ldots\right)+\sum_{i \geqslant 2} \frac{1}{i} Z_{\left(\mathcal{B}^{\prime}\right)^{\iota}}\left(\tilde{\mathscr{A}}^{\omega^{i}}\left(z^{i}\right), \tilde{\mathscr{A}}^{\omega^{2 i}}\left(z^{2 i}\right), \ldots\right)\right) .
$$

For $d=1$, it follows for example by [36, Lem. 3.2] that

$$
\left[z^{n}\right] \tilde{\mathscr{G}}^{\omega}(z) \sim \tilde{\mathscr{G}}^{\omega}\left(\rho_{\mathscr{A}}\right)\left[z^{n}\right] \tilde{\mathscr{C}}^{\omega}(z)
$$

with $\tilde{\mathscr{G}}^{\omega}\left(\rho_{\mathscr{A}}\right)=\exp \left(\sum_{i \geqslant 1} \tilde{\mathscr{C}}^{\omega}\left(\rho_{\mathscr{A}}^{i}\right) / i\right)$. Hence we recover the asymptotic expansion obtained in [15, Thm. 15]. By [36, Thm. 3.1] it follows that the largest connected component of the random graph $\mathrm{G}_{n}^{\omega}$ has size $n+O_{p}(1)$, and that the remaining small fragments $\operatorname{frag}\left(\mathrm{G}_{n}^{\omega}\right)$ satisfy the limit

$$
\operatorname{frag}\left(\mathrm{G}_{n}^{\omega}\right) \stackrel{d}{\longrightarrow} \mathrm{G}_{0}
$$

with $\mathrm{G}_{0}$ defined in Theorem 5 .

In the general case $d \geqslant 1$, the modulo of $n$ imposes restrictions on the number of components, as connected components whose number of vertices does not lie in $1+d \mathbb{N}_{0}$ have weight zero. Thus for $n \equiv a \bmod d, 0 \leqslant a<d$ an $n$-sized unlabelled graph from $\mathscr{G}^{\omega}$ with non-zero weight is a multiset of connected unlabelled graphs with the total number of elements belonging to $a+d \mathbb{N}_{0}$. (The converse is ensured to hold when all connected components are sufficiently large, since there are only finitely many unlabelled connected graphs with size in $1+d \mathbb{N}_{0}$ and weight zero.) We let $\mathrm{SET}_{a}$ denote the species with a single unlabelled object of size $k$ for each $k \in a+d \mathbb{N}_{0}$. It is easy to generalize [36, Thm. 3.1, Lem. 3.2] to obtain

$$
\left[z^{n}\right] \tilde{\mathscr{G}}^{\omega}(z) \sim Z_{\operatorname{SET}_{a}^{\prime}}\left(\tilde{\mathscr{A}}^{\omega}\left(\rho_{\mathscr{A}}\right), \tilde{\mathscr{A}}^{\omega^{2}}\left(\rho_{\mathscr{A}}^{2}\right), \ldots\right)\left[z^{n+1-a}\right] \tilde{\mathscr{C}}^{\omega}(z) \quad \text { and } \quad \operatorname{frag}\left(\mathrm{G}_{n}^{\omega}\right) \stackrel{d}{\longrightarrow} \mathrm{G}_{a}
$$

as $n$ tends to infinity along the lattice $a+d \mathbb{N}_{0}$, with $\mathrm{G}_{a}$ the random graph defined in Theorem 5. (Compare with [32, Thm. 3.4], where such a generalization was carried out in the labelled setting.) This finalizes the proof of Theorem 5 .

\section{Approximating unrooted graphs by rooted graphs (Proof of Theorem 1)}

Throughout we assume that (2) holds.

Suppose that $\bar{Z}_{\left(\left(\mathscr{B}^{\prime}\right) \circledast\right)^{\imath}} \neq 0$. In order to prove Theorem 1 it suffices by Decompositions (19) and (20) to show such approximation statements for random unlabelled $n$-vertex graphs sampled with probability proportional to their weight from the classes $\mathscr{A}^{\omega},\left(\mathscr{C}_{v}^{\circledast}\right)^{\omega}$ and $\left(\mathscr{C}_{b}^{\circledast}\right)^{\omega}$. For the class $\mathscr{A}^{\omega}$ this is trivial, and for the other two classes this is precisely what we did in Lemma 7 and Lemma 10. Hence Theorem 1 holds in this case, and we 
even obtain that $\mathrm{C}_{n}^{\omega} \simeq \mathrm{D}_{n}+\mathrm{A}_{n-d_{n}}^{\omega}$. That is, the upper bound for the total variational distance in Inequality (3) is equal to zero.

In the case $\bar{Z}_{\left(\left(\mathscr{B}^{\prime}\right)^{\circledast}\right)^{\iota}}=0$ we may argue again that analogous approximations as in Theorem 1 hold for the classes $\mathscr{A}^{\omega}$ and $\left(\mathscr{C}_{v}^{\circledast}\right)^{\omega}$. However, such a statement does not appear to hold any longer for the class $\left(\mathscr{C}_{b}^{\circledast}\right)^{\omega}$. This is not a problem, as Lemma 9 and Equation (37) guarantee that a random $n$-vertex cycle-pointed connected graph sampled with probability proportional to its $\omega$-weight belongs only with exponentially small probability to the class $\left(\mathscr{C}_{b}^{\circledast}\right)^{\omega}$. Hence Theorem 1 follows, and Inequality (3) holds with the upper bound of the total variational distance being given by the quotient $\frac{\left[z^{n}\right]\left(\tilde{\mathscr{C}}^{\circledast}\right)^{\omega}(z)}{n\left[z^{n}\right] \tilde{\mathscr{C}}^{\omega}(z)} \leqslant C \exp (-c n)$ uniformly in all $n$ for fixed constants $C, c>0$ that do not depend on $n$.

\section{$5 \quad$ Scaling limit (Proof of Theorem 2)}

\subsection{Background on the Gromov-Hausdorff-Prokhorov metric}

We recall relevant notions for random measured metric spaces. Most parts of the present exposition follow [28, Sec. 6]. Given two compact subsets $K_{1}, K_{2}$ of a metric space $\left(X, d_{X}\right)$, we may consider their Hausdorff distance

$$
d_{\mathrm{H}}\left(K_{1}, K_{2}\right)=\inf \left\{\epsilon>0 \mid K_{2} \subset U_{\epsilon}\left(K_{1}\right), K_{1} \subset U_{\epsilon}\left(K_{2}\right)\right\} .
$$

Here $U_{\epsilon}(K):=\left\{x \in X \mid d_{X}(x, K)<\epsilon\right\}$ denotes the $\epsilon$-thickening of a subset $K \subset X$. The Prokhorov distance between two Borel probability measures $P_{1}, P_{2}$ on $X$ is defined by

$$
\begin{aligned}
& d_{\mathrm{H}}\left(P_{1}, P_{2}\right) \\
& =\inf \left\{\epsilon>0 \mid \text { for all } A \subset X \text { closed: } P_{1}(A) \leqslant P_{2}\left(U_{\epsilon}(A)\right)+\epsilon, P_{2}(A) \leqslant P_{1}\left(U_{\epsilon}(A)\right)+\epsilon\right\} .
\end{aligned}
$$

Let $\left(X, d_{X}, P_{X}\right),\left(Y, d_{Y}, P_{Y}\right)$ be compact metric spaces equipped with Borel probability measures. For any metric space $\left(E, d_{E}\right)$ and isometric embeddings $\iota_{X}: X \rightarrow E$ and $\iota_{Y}: Y \rightarrow E$ we may consider the push-forward measures $P_{X} \iota_{X}^{-1}$ and $P_{Y} \iota_{Y}^{-1}$. The GromovHausdorff-Prokhorov (GHP) distance between the two spaces is given by

$$
d_{\mathrm{GHP}}\left(\left(X, d_{X}, P_{X}\right),\left(Y, d_{Y}, P_{Y}\right)\right)=\inf _{\left(E, d_{E}\right), \iota_{X}, \iota_{Y}} \min \left(d_{\mathrm{H}}\left(\iota_{X}(X), \iota_{Y}(Y)\right), d_{\mathrm{P}}\left(P_{X} \iota_{X}^{-1}, P_{Y} \iota_{Y}^{-1}\right)\right),
$$

with the index ranging over all possible isometric embeddings $\iota_{X}, \iota_{Y}$ of $X$ and $Y$ into any possible common metric space $\left(E, d_{E}\right)$.

The GHP distance satisfies the axioms of a premetric on the collection of compact metric spaces equipped with Borel probability measures. The corresponding metric on the quotient space $\mathbb{K}$ is complete and separable. That is, $\mathbb{K}$ is a Polish space. For settheoretic reasons, we would actually have to work with a set of representatives instead of a collection of proper class, but this a purely notational issues that we may safely ignore.

We are usually not going to distinguish between a measured compact metric space and the corresponding equivalence class. Also, whenever there is no risk of confusion, we 
will write $\lambda X$ instead of $\left(X, \lambda d_{X}, P_{X}\right)$ for any scalar factor $\lambda>0$ and any compact metric space $\left(X, d_{X}\right)$ equipped with a Borel probability measure $P_{X}$.

If we distinguish points $x_{0} \in X$ and $y_{0} \in Y$ we may also form the rooted GromovHausdorff-Prokhorov-distance $d_{\mathrm{GHP}}^{c}\left(X^{\bullet}, Y^{\bullet}\right)$ between the rooted spaces $X^{\bullet}=\left(X, x_{0}\right)$ and $Y^{\bullet}=\left(Y, y_{0}\right)$ by

$$
\inf _{\left(E, d_{E}\right), \iota_{X}, \iota_{Y}} \min \left(d_{\mathrm{H}}\left(\iota_{X}(X), \iota_{Y}(Y)\right), d_{\mathrm{P}}\left(P_{X} \iota_{X}^{-1}, P_{Y} \iota_{Y}^{-1}\right), d_{E}\left(\iota_{X}\left(x_{0}\right), \iota_{Y}\left(y_{0}\right)\right)\right) .
$$

The rooted GHP-distance $d_{\mathrm{GHP}}^{c}$ satisfies analogous properties as $d_{\mathrm{GHP}}$, see [1, Thm. 2.3] for details.

\subsection{Scaling limits of random unlabelled unrooted graphs}

We first prove the convergence stated in (4). Recall the following scaling limit for the random rooted graph $A_{n}^{\omega}$.

Lemma 11 ([33, Thm. 33]). Suppose that the tree-like Assumption (2) is satisfied. Then there exists a constant $c_{\omega}>0$ such that the random rooted graph $\mathrm{A}_{n}^{\omega}$ equipped with the uniform measure $\mu_{n}^{\mathcal{A}}$ on its set of vertices satisfies the limit

$$
\left(\mathrm{A}_{n}^{\omega}, \frac{c_{\omega}}{\sqrt{n}} d_{\mathrm{A}_{n}^{\omega}}, \mu_{n}^{\mathscr{A}}\right) \stackrel{d}{\longrightarrow}\left(\mathcal{T}_{\mathrm{e}}, d_{\mathcal{T}_{\mathrm{e}}}, \mu\right)
$$

in the rooted Gromov-Hausdorff-Prokhorov sense. The diameter $\mathbf{D}\left(\mathrm{A}_{n}^{\omega}\right)$ satisfies the tail bound

$$
\mathbb{P}\left(\mathbf{D}\left(\mathrm{A}_{n}^{\omega}\right) \geqslant x\right) \leqslant C \exp \left(-c x^{2} / n\right)
$$

To be precise, [33] actually states Gromov-Hausdorff convergence of $\mathrm{A}_{n}^{\omega}$ to the Brownian tree, but it is not hard to see that the arguments may be extended to obtain GromovHausdorff-Prokhorov convergence.

As the graph $\mathrm{D}_{n}$ from Theorem 1 has size $d_{n}=O_{p}(1)$ it follows from Lemma 11 that

$$
\left(\mathrm{A}_{n-d_{n}}^{\omega}, \frac{c_{\omega}}{\sqrt{n}} d_{\mathrm{A}_{n-d_{n}}^{\omega}}, \mu_{n-d_{n}}^{\mathscr{A}}\right) \stackrel{d}{\longrightarrow}\left(\mathcal{T}_{\mathrm{e}}, d_{\mathcal{T}_{\mathrm{e}}}, \mu\right)
$$

Let $\mu_{n}^{\mathscr{A}+\mathscr{D}}$ denote the uniform measure on the vertex set of the graph $\mathrm{A}_{n}^{\omega}+\mathrm{D}_{n}$. Using again $d_{n}=O_{p}(1)$ we may observe

$$
d_{\mathrm{GHP}}\left(\left(\mathrm{A}_{n-d_{n}}^{\omega}+\mathrm{D}_{n}, \frac{c_{\omega}}{\sqrt{n}} d_{\mathrm{A}_{n-d_{n}}^{\omega}+\mathrm{D}_{n}}, \mu_{n}^{\mathscr{A}+\mathscr{D}}\right),\left(\mathrm{A}_{n-d_{n}}^{\omega}, \frac{c_{\omega}}{\sqrt{n}} d_{\mathrm{A}_{n-d_{n}}^{\omega}}, \mu_{n-d_{n}}^{\mathscr{A}}\right)\right) \stackrel{p}{\longrightarrow} 0 .
$$

By Theorem 1, it follows that

$$
\left(\mathrm{C}_{n}^{\omega}, \frac{c_{\omega}}{\sqrt{n}} d_{\mathrm{C}_{n}^{\omega}}, \mu_{n}\right) \stackrel{d}{\longrightarrow}\left(\mathcal{T}_{\mathrm{e}}, d_{\mathcal{T}_{\mathrm{e}}}, \mu\right)
$$


This verifies the limit (4).

In order to complete the proof of Theorem 2 , it remains to prove Inequality (5). To this end, it suffices to verify (5) uniformly for $\sqrt{n} \leqslant x \leqslant n$. Indeed, the diameter of $C_{n}^{\omega}$ is at most $n$, hence the left hand side of (5) equals zero when $x>n$. In this case, (5) is trivially satisfied. Furthermore, by possibly replacing $C$ by a large value and $c$ by a smaller value, we may always assume $C>1 / e$ and $c<1$ so that so that for all $x<\sqrt{n}$ we have $C \exp \left(-c x^{2} / n\right) \geqslant C \exp (-c)>1$. Thus, (5) is trivially satisfied in this case.

Moreover, we may treat the three individual parts of the decomposition

$$
\left(\mathscr{C}^{\circ}\right)^{\omega} \simeq \mathscr{A}^{\omega}+\left(\mathscr{C}_{v}^{\circledast}\right)^{\omega}+\left(\mathscr{C}_{b}^{\circledast}\right)^{\omega}
$$

in (19) and (20) individually. Inequality (44) takes care of the first summand $\mathscr{A}^{\omega}$. As for the case of a vertex cycle-center, (23) allows us to sample an $n$-vertex unlabelled graph from $\left(\mathscr{C}_{v}^{\circledast}\right)^{\omega}$ with probability proportional to its weight by conditioning the following procedure on producing a graph with size $n$ :

1. Draw a random unlabelled $\mathscr{A}^{\omega}$-object A with probability

$$
\mathbb{P}(\mathrm{A}=A)=\omega(A) \rho_{\mathscr{A}}^{|A|} / \tilde{\mathscr{A}}^{\omega}\left(\rho_{\mathscr{A}}\right) .
$$

2. Draw a random unlabelled $\mathscr{P} \odot\left(\left(\mathscr{B}^{\prime}\right)^{\iota} \circ \mathscr{A}^{\omega}\right)$-object $\mathrm{P}$ with $\mathbb{P}(\mathrm{P}=P)$ proportional to $\omega(P) \rho_{\mathscr{A}}^{|P|}$.

3. Glue $\mathbf{A}$ and $\mathbf{P}$ together at their root vertices to form the graph $A+P$.

Note that the total size of the graph $A+P$ is $|A|+|P|-1$ as we identify the two roots. By (25) and (28) we know that

$$
\mathbb{P}(|\mathrm{A}|+|\mathrm{P}|-1=n)=\rho_{\mathscr{A}}^{n}\left[z^{n}\right]\left(\tilde{\mathscr{C}}_{v}^{\circledast}\right)^{\omega}(z) /\left(\tilde{\mathscr{C}}_{v}^{\circledast}\right)^{\omega}\left(\rho_{\mathscr{A}}\right)=O\left(n^{3 / 2}\right) .
$$

If $\mathbf{D}(\mathrm{A}+\mathrm{P}) \geqslant x$ then it holds that $\mathbf{D}(\mathrm{A}) \geqslant x / 2$ or $|\mathrm{P}| \geqslant x / 2$. It follows that

$$
\begin{aligned}
\mathbb{P}(\mathbf{D}(\mathrm{A}+\mathrm{P}) \geqslant x|| \mathrm{A} \mid & +|\mathrm{P}|=n-1) \\
& \leqslant \mathbb{P}(\mathbf{D}(\mathrm{A}) \geqslant x / 2|| \mathrm{A}|+| \mathrm{P} \mid=n-1)+O\left(n^{3 / 2}\right) \mathbb{P}(|\mathrm{P}| \geqslant x / 2) .
\end{aligned}
$$

By Lemma 6 there are constants $C^{\prime}, c^{\prime}>0$ such that

$$
\mathbb{P}(|\mathrm{P}| \geqslant y) \leqslant C^{\prime} \exp \left(-c^{\prime} y\right)
$$

for all $y$. It follows that uniformly for all $\sqrt{n} \leqslant x \leqslant n$ we have

$$
\begin{aligned}
O\left(n^{3 / 2}\right) \mathbb{P}(|\mathrm{P}| \geqslant x / 2) & \leqslant O\left(n^{3 / 2}\right) \exp \left(-c^{\prime} x / 2\right) \\
& =\exp \left(-c^{\prime}(1+o(1)) x / 2\right) \\
& \leqslant \exp \left(-c^{\prime \prime} x^{2} / n\right)
\end{aligned}
$$


for some fixed constant $c^{\prime \prime}>0$. Using (44) and $x \leqslant n$ we obtain also that

$$
\begin{aligned}
& \mathbb{P}(\mathbf{D}(\mathrm{A}) \geqslant x / 2|| \mathrm{A}|+| \mathrm{P} \mid=n-1) \\
& \leqslant \sum_{k=x / 2}^{n} \mathbb{P}(\mathbf{D}(\mathrm{A}) \geqslant x / 2|| \mathrm{A} \mid=k) \mathbb{P}(|\mathrm{A}|=k|| \mathrm{A}|+| \mathrm{P} \mid=n-1) \\
& \leqslant C \sum_{k=x / 2}^{n} \exp \left(-c x^{2} /(4 k)\right) \mathbb{P}(|\mathrm{A}|=k|| \mathrm{A}|+| \mathrm{P} \mid=n-1) \\
& \leqslant C \exp \left(-c x^{2} /(4 n)\right) .
\end{aligned}
$$

Combining (45), (46) and (47) it follows that

$$
\mathbb{P}(\mathbf{D}(\mathrm{A}+\mathrm{P}) \geqslant x|| \mathrm{A}|+| \mathrm{P} \mid=n-1) \leqslant C^{\prime \prime \prime} \exp \left(-c^{\prime \prime \prime} x^{2} / n\right)
$$

uniformly for $\sqrt{n} \leqslant x \leqslant n$ for some constants $C^{\prime \prime \prime}, c^{\prime \prime \prime}>0$.

It remains to verify such a bound for the case of a block cycle-center. By Lemma 9 it follows that in case $\bar{Z}_{\left(\left(\mathscr{B}^{\prime}\right)^{\circledast}\right)^{\iota}}=0$ the probability for a random unlabelled $n$-vertex graph from the class $\left(\mathscr{C}^{\circ}\right)^{\omega}$ have a block cycle-center is exponentially small, that is, it is bounded by $C_{1} \exp \left(-c_{1} n\right)$ from some constants $C_{1}, c_{1}>0$ that do not depend on $n$. As $x \leqslant n$ we have

$$
C_{1} \exp \left(-c_{1} n\right) \leqslant C_{1} \exp \left(-c_{1} x^{2} / n\right)
$$

and hence we are done in this case.

In case $\bar{Z}_{\left(\left(\mathscr{B}^{\prime}\right)^{\circledast}\right)^{\iota}} \neq 0$ the strategy is similar to the case of a vertex cycle-center, but the details are more technical. Recall the random graph $C$ from Equation (32) and its sampling procedure in subsequent paragraphs that splits it into a part with $H$ vertices and $F$ rooted components $\mathrm{A}_{1}, \ldots, \mathrm{A}_{F}$ as in (34). If $\mathbf{D}(\mathbf{C}) \geqslant x$ then it holds that $H \geqslant x / 2$ or $\max \left(\mathbf{D}\left(\mathrm{A}_{1}\right), \ldots, \mathbf{D}\left(\mathrm{A}_{F}\right)\right) \geqslant x / 4$. As $\mathbb{P}(|\mathrm{C}|=n)=O\left(n^{3 / 2}\right)$ by (35) and (25), it follows that

$$
\mathbb{P}(\mathbf{D}(\mathrm{C})|| \mathrm{C} \mid=n) \leqslant \mathbb{P}\left(\max \left(\mathbf{D}\left(\mathrm{A}_{1}\right), \ldots, \mathbf{D}\left(\mathrm{A}_{F}\right)\right) \geqslant x / 4|| \mathrm{C} \mid=n\right)+O\left(n^{3 / 2}\right) \mathbb{P}(H \geqslant x / 2) .
$$

Lemma 8 ensures that the vector $(F, H)$ has finite exponential moments. In particular there are constants $C_{1}^{\prime}, c_{1}^{\prime}>0$ such that $\mathbb{P}(H \geqslant y) \leqslant C_{1}^{\prime} \exp \left(-c_{1}^{\prime} y\right)$ uniformly for all $y$. Hence

$$
\begin{aligned}
O\left(n^{3 / 2}\right) \mathbb{P}(H \geqslant x / 2) & \leqslant O\left(n^{3 / 2}\right) \exp \left(-c_{1}^{\prime} x / 2\right) \\
& =O(1) \exp \left(-c_{1}^{\prime}(1+o(1)) x / 2\right) \\
& \leqslant C_{1}^{\prime \prime} \exp \left(-c_{1}^{\prime \prime} x^{2} / n\right)
\end{aligned}
$$

for some constants $C_{1}^{\prime \prime}, c_{1}^{\prime \prime}>0$. As for the other summand in (48), for any $f^{\prime}, k_{1}, \ldots, k_{f^{\prime}} \geqslant 0$ let $\mathcal{E}$ denote the event that $F=f^{\prime}$ and $\left|\mathrm{A}_{i}\right|=k_{i}$ for all $1 \leqslant i \leqslant f^{\prime}$. We may argue using 
(44) that

$$
\begin{aligned}
\mathbb{P}\left(\max _{1 \leqslant i \leqslant F} \mathbf{D}\left(\mathrm{A}_{i}\right) \geqslant x / 4|| \mathrm{C} \mid=n\right) & \leqslant \sum_{1 \leqslant f^{\prime}, k_{1}, \ldots, k_{f^{\prime}} \leqslant n} \mathbb{P}\left(\max _{1 \leqslant i \leqslant F} \mathbf{D}\left(\mathrm{A}_{i}\right) \geqslant x / 4 \mid \mathcal{E}\right) \mathbb{P}(\mathcal{E}|| \mathrm{C} \mid=n) \\
& \leqslant \sum_{1 \leqslant f^{\prime}, k_{1}, \ldots, k_{f^{\prime}} \leqslant n} \sum_{1 \leqslant i \leqslant f^{\prime}} C \exp \left(-c x^{2} /\left(16 k_{i}\right)\right) \mathbb{P}(\mathcal{E}|| \mathrm{C} \mid=n) \\
& \leqslant C \exp \left(-c x^{2} /(16 n)\right) \sum_{1 \leqslant f^{\prime}, k_{1}, \ldots, k_{f^{\prime}} \leqslant n} f^{\prime} \mathbb{P}(\mathcal{E}|| \mathrm{C} \mid=n) \\
& \leqslant C \exp \left(-c x^{2} /(16 n)\right) \mathbb{E}[F|| \mathrm{C} \mid=n] .
\end{aligned}
$$

It follows from the expression (33) of the joint probability generating function of $F$ and $H$ that

$$
\mathbb{E}[F|| C \mid=n]=\frac{\left[z^{n}\right] \frac{\partial f}{\partial x}\left(\tilde{\mathscr{A}}^{\omega}\left(z \rho_{\mathscr{A}}\right), z \rho_{\mathscr{A}}\right)}{\left[z^{n}\right] f\left(\tilde{\mathscr{A}}^{\omega}\left(z \rho_{\mathscr{A}}\right), z \rho_{\mathscr{A}}\right)} .
$$

We know by (36) that $\left[z^{n}\right] f\left(\tilde{\mathscr{A}}^{\omega}\left(z \rho_{\mathscr{A}}\right), z \rho_{\mathscr{A}}\right)$ is asymptotically equivalent to $\left[z^{n}\right] \tilde{\mathscr{A}}^{\omega}(z)$ up to a constant factor. By the same arguments (just with $\frac{\partial f}{\partial x}$ instead of $f$ ) the same holds for $\left[z^{n}\right] \frac{\partial f}{\partial x}\left(\tilde{A}^{\omega}\left(z \rho_{\mathscr{A}}\right), z \rho_{\mathscr{A}}\right)$. (This could also be verified using the usual singularity analysis methods from [19, Thm. VI.5].) Consequently, the conditional expectation $\mathbb{E}[F|| C \mid=n]$ remains bounded as $n$ tends to infinity. It follows from this fact and Inequalities (48), (49), and (50) that

$$
\mathbb{P}(\mathbf{D}(\mathrm{C})|| \mathrm{C} \mid=n) \leqslant C_{1}^{\prime \prime} \exp \left(-c_{1}^{\prime \prime} x^{2} / n\right)
$$

holds uniformly in $\sqrt{n} \leqslant x \leqslant n$ for some constants $C_{1}^{\prime \prime}, c_{1}^{\prime \prime}>0$. This completes the proof of Theorem 2.

\section{Local limit (Proof of Theorem 4)}

\subsection{Local topology}

We recall relevant properties of the local topology.

Given two connected, rooted, and locally finite graphs $G^{\bullet}=\left(G, v_{G}\right)$ and $H^{\bullet}=\left(H, v_{H}\right)$ we may consider their distance

$$
d_{\mathrm{loc}}\left(G^{\bullet}, H^{\bullet}\right)=2^{-\sup \left\{k \in \mathbb{N}_{0} \mid U_{k}\left(G^{\bullet}\right) \simeq U_{k}\left(H^{\bullet}\right)\right\}}
$$

with $U_{k}\left(G^{\bullet}\right) \simeq U_{k}\left(H^{\bullet}\right)$ denoting isomorphism of rooted graphs. This defines a premetric on the collection of all rooted locally finite connected graphs. Two such graphs have distance zero, if and only if they are isomorphic. Hence we obtain a metric on the collection $\mathbb{B}$ of all unlabelled, connected, rooted, locally finite graphs. There are some set-theoretic caveats that actually require us to work with a set of representatives instead of a collection of proper classes, but we may safely ignore this purely notational issue.

Weak convergence of a sequence $\left(\mathrm{G}_{n}, v_{n}\right)_{n \geqslant 1}$ of random pointed graphs in $\mathbb{B}$ is also called local weak convergence. In the special case where for each $n$ the random graph $\mathrm{G}_{n}$ is almost surely finite, and the root $v_{n}$ is selected uniformly at random from its vertices, it is also called distributional or Benjamini-Schramm convergence. 


\subsection{Local convergence of random unlabelled unrooted graphs}

It was shown in [33, Thm. 32] that there exists a random rooted graph $\hat{\mathrm{C}}$ such that for any sequence $k_{n}=o(\sqrt{n})$ the $k_{n}$ neighbourhood $U_{k_{n}}\left(\mathrm{~A}_{n}^{\omega}, u_{n}\right)$ of a uniformly selected vertex $u_{n} \in \mathrm{A}_{n}^{\omega}$ satisfies

$$
d_{\mathrm{TV}}\left(U_{k_{n}}\left(\mathrm{~A}_{n}^{\omega}, u_{n}\right), U_{k_{n}}(\hat{\mathrm{C}})\right) \rightarrow 0 .
$$

This was obtained from a more general result [33, Thm. 27], that also yields that the root of $\mathrm{A}_{n}^{\omega}$ is with high probability not contained in $U_{k_{n}}\left(\mathrm{~A}_{n}^{\omega}, u_{n}\right)$. By Theorem 1 we know that a uniformly selected vertex $x_{n}$ of $\mathrm{D}_{n}+\mathrm{A}_{n-d_{n}}^{\omega}$ lies with high probability in $\mathrm{A}_{n-d_{n}}^{\omega}$, since $\mathrm{D}_{n}$ accounts for stochastically bounded subset of the $n$ vertices. Conditioned on this event, the vertex $x_{n}$ is uniformly distributed among the vertices of $\mathrm{A}_{n-d_{n}}^{\omega}$. Since $k_{n} / \sqrt{n-d_{n}}=o_{p}(1)$, it follows that with high probability the $k_{n}$ neighbourhood of $x_{n}$ does not contain the root of $\mathrm{A}_{n-d_{n}}^{\omega}$. That is, $U_{k_{n}}\left(\mathrm{D}_{n}+\mathrm{A}_{n-d_{n}}^{\omega}, x_{n}\right)=U_{k_{n}}\left(\mathrm{~A}_{n-d_{n}}^{\omega}, x_{n}\right)$ holds with probability tending to 1 as $n$ becomes large. It follows from (3) that the uniformly selected vertex $v_{n} \in \mathrm{C}_{n}^{\omega}$ satisfies

$$
d_{\mathrm{TV}}\left(U_{k_{n}}\left(\mathrm{C}_{n}^{\omega}, u_{n}\right), U_{k_{n}}(\hat{\mathrm{C}})\right) \rightarrow 0 .
$$

This proves Theorem 4.

\section{The scaling constant of unlabelled outerplanar graphs}

In this section, we provide a proof of Theorem 3.

\subsection{A general description}

The scaling constant $c_{\omega}$ of Theorem 2 is identical to the scaling constant for unlabelled rooted graphs in Lemma 11. Hence, by the general result [33, Lem. 30, Proof of Thm. 28 ], it is given by

$$
c_{\omega}=\frac{\sqrt{(1+\mathbb{E}[\zeta]) \mathbb{V}[\xi]}}{2 \mathbb{E}[\eta]}
$$

with $\xi$ and $\zeta$ the random non-negative integers with probability generating function

$$
\mathbb{E}\left[z^{\xi} w^{\zeta}\right]=\exp \left(z \tilde{\mathscr{A}}^{\omega}\left(\rho_{\mathscr{A}}\right)+\sum_{i=2}^{\infty} \tilde{\mathscr{A}}^{i}\left(\rho_{\mathscr{A}}^{i} w^{i}\right)\right) \rho_{\mathscr{A}} / \tilde{\mathscr{A}}^{\omega}\left(\rho_{\mathscr{A}}\right),
$$

and $\eta$ the distance between the marked points in a random unlabelled $\left(\mathscr{B}^{\prime \bullet}\right)^{\iota}$-object $\mathrm{B}^{\prime \bullet}$ that follows a Boltzmann distribution with parameter $\tilde{\mathscr{A}}^{\omega}\left(\rho_{\mathscr{A}}\right)$. That is, $\mathrm{B}^{\prime \bullet}$ is equal to a random unlabelled $\left(\mathscr{B}^{\prime \bullet}\right)^{\iota}$-object $B$ with probability $\tilde{\mathscr{A}}^{\omega}\left(\rho_{\mathscr{A}}\right)^{|B|} \iota(B) /\left(\tilde{\mathscr{B}}^{\prime \bullet}\right)^{\iota}\left(\tilde{\mathscr{A}}^{\omega}\left(\rho_{\mathscr{A}}\right)\right)$. Note that $B$ is a graph having an inner root (also called the $*$-vertex) that does not contribute to the total size $|B|$, and an outer root that does contribute to the total size 
and is required to not coincide with the inner root. It follows from the expression for the generating function of $(\xi, \zeta)$ in Equation (53) that

$$
\mathbb{V}[\xi]=E_{u u}\left(\rho_{\mathscr{A}}, \tilde{\mathscr{A}}^{\omega}\left(\rho_{\mathscr{A}}\right)\right) \tilde{\mathscr{A}}^{\omega}\left(\rho_{\mathscr{A}}\right) \quad \text { and } \quad \mathbb{E}[\zeta]=E_{z}\left(\rho_{\mathscr{A}}, \tilde{\mathscr{A}}^{\omega}\left(\rho_{\mathscr{A}}\right)\right) \rho_{\mathscr{A}} / \tilde{\mathscr{A}}^{\omega}\left(\rho_{\mathscr{A}}\right)-1,
$$

with the power series $E(z, u)$ defined in Equation (39) (and $E_{z}$ and $E_{u u}$ denoting partial derivatives). The main challenge is to compute $\mathbb{E}[\eta]$.

\subsection{Unlabelled outerplanar graphs}

We are now going to derive numeric approximations of the constant $c_{\omega}$ in (52) for the $\omega$-weighting $\omega_{\mathscr{O}}$ that corresponds to uniform unlabelled outerplanar graphs. It was shown in [37, Thm. 2.16], [9, Cor. 3.6] that

$$
\begin{aligned}
& Z_{\left(\mathscr{B}^{\prime}\right)^{\iota} \mathscr{O}}\left(s_{1}, s_{2}, \ldots\right)= \\
& \quad \frac{1}{8}\left(1+s_{1}-\sqrt{s_{1}^{2}-6 s_{1}+1}\right)+\frac{1}{8 s_{2}^{2}}\left(s_{1}+s_{2}\right)\left(1-3 s_{2}-\sqrt{s_{2}^{2}-6 s_{2}+1}\right),
\end{aligned}
$$

with $\iota_{\mathscr{O}}$ denoting the special case of the $\iota$-weighting for outerplanar graphs. The formula was obtained in the cited sources by computing $Z_{(\mathscr{B})^{\iota} \mathcal{O}}$ and then using $Z_{(\mathscr{B})^{\iota} \mathscr{O}}=\frac{\partial}{\partial s_{1}} Z_{(\mathscr{B})^{\iota} \mathcal{O}}$. We are going to derive (55) in a direct way that will be convenient later on for the computation of $c_{\omega}$.

To this end, consider the species $\mathscr{D}$ of dissections of polygons where one edge lying on the frontier of the outer face is marked and oriented, and where the origin of the root-edge is a $*$-vertex that does not contribute to the total size. Note that $\mathscr{D}$ is, like any corner-rooted planar map, asymmetric, meaning that any permutation of the non-*-vertices that leaves the structure invariant must be the identity. The smallest $\mathscr{D}$-object has size 1 and consists of a single oriented root-edge. Any larger $\mathscr{D}$-object may

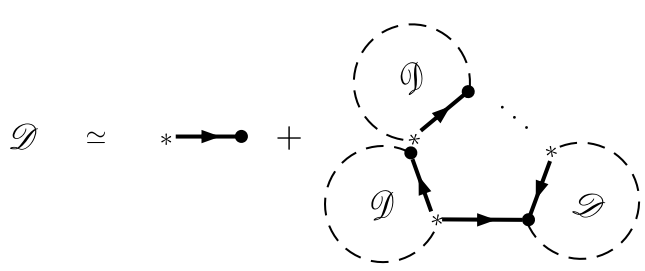

Figure 4: Decomposition of dissections be decomposed in a unique way into an ordered list of at least two $\mathscr{D}$-objects as illustrated in Figure 4, yielding

$$
\mathscr{D} \simeq \mathscr{X}+\sum_{k \geqslant 2} \mathscr{D}^{k} \quad \text { and } \quad Z_{\mathscr{D}}=s_{1}+Z_{\mathscr{D}}^{2} /\left(1-Z_{\mathscr{D}}\right)=\frac{1}{4}\left(1+s_{1}-\sqrt{s_{1}^{2}-6 s_{1}+1}\right) .
$$

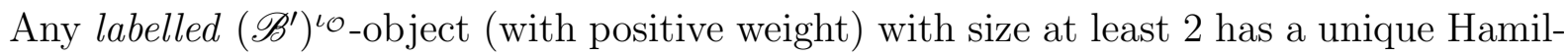
ton cycle that may be oriented in two different ways, yielding

$$
Z_{0}:=Z_{\left(\mathscr{B}^{\prime}\right)^{\iota} \mathscr{O}}\left(s_{1}, 0,0, \ldots\right)=\frac{1}{2}\left(Z_{\mathscr{D}}+s_{1}\right) .
$$


It remains to sum up the weight-monomials of symmetries that are different from the

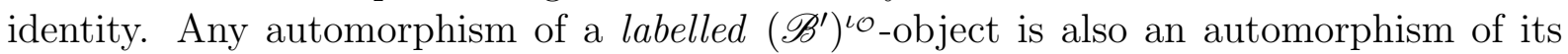
Hamilton cycle and hence an element of a dihedral group, meaning it is composed out of rotations and reflections along axis passing through the "center" of the Hamilton cycle. But the *-vertex is always required to be fixed, hence the only possible non-trivial automorphism is a reflection along the axis that passes through the $*$-vertex and the "center". This also means that any such graph has only two proper embeddings into the plane, so it makes sense to define the root-face as the unique inner face adjacent to the $*$-root.

There are two different cases, depending on whether the

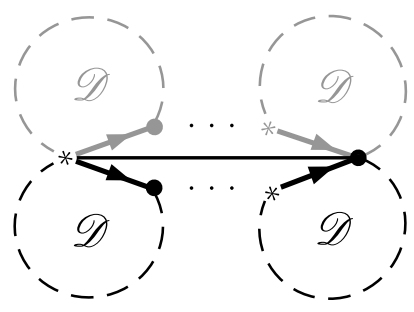

Figure 5: Symmetric blocks axis leaves the Hamilton cycle through the middle of an edge, or through a non-*-vertex. The latter case may be partitioned into two subcases, depending on whether there exists an edge between the $*$-vertex and this second vertex or not. If this edge is present, then the graph is uniquely determined by the ordered list of $\mathscr{D}$-objects encountered along one half of the root-face, see Figure 5. This list must have length at least two as we do not allow multi-edges. All atoms belong to 2-cycles of the reflection, except for the destination of the root-edge in the last element of the list, since this vertex must be a fixed-point. Moreover, any such graph with $n$ non*-vertices has precisely $n ! / 2$ labellings, so the sum of weight-monomials of all symmetries of such dissections is given by

$$
Z_{1}:=\frac{1}{2}\left(\sum_{k \geqslant 2} \mathscr{D}\left(s_{2}\right)^{k}\right) \frac{s_{1}}{s_{2}}=\frac{\mathscr{D}\left(s_{2}\right)^{2} \frac{s_{1}}{s_{2}}}{2\left(1-\mathscr{D}\left(s_{2}\right)\right)}=\frac{1}{2}\left(\mathscr{D}\left(s_{2}\right) \frac{s_{1}}{s_{2}}-s_{1}\right) .
$$

with $\mathscr{D}(z):=Z_{\mathscr{D}}(z, 0,0, \ldots)$.

If there exists no edge along the axis of symmetry, then the endpoints of the root-edges of any corresponding pair of identical dissections may be joined by chord. Compare with the upper part of Figure 6, where these potential chords are indicated by dashed vertical straight lines. Again, any unlabelled graph of this form with $n$ non-*-vertices has precisely $n ! / 2$ labellings. So the cycle index sums for the case where the axis passes through two vertices is given by

$$
Z_{2}:=\frac{1}{2}\left(\sum_{k \geqslant 1}\left(2 \mathscr{D}\left(s_{2}\right)\right)^{k}\right) \mathscr{D}\left(s_{2}\right) \frac{s_{1}}{s_{2}}=\frac{\mathscr{D}\left(s_{2}\right)^{2} \frac{s_{1}}{s_{2}}}{1-2 \mathscr{D}\left(s_{2}\right)} .
$$

Here the factor 2 in front of $Z_{\mathscr{D}}$ is due to the two options that the chord is present or not. Similarly, the case where the axis

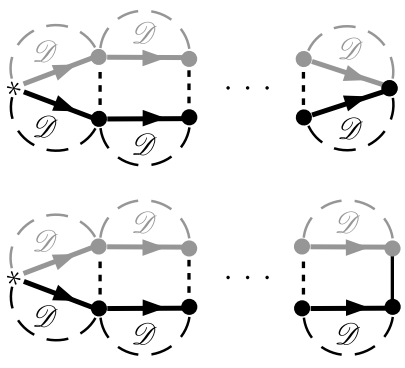

Figure 6: Symmetric derived blocks with no edge along the axis of symmetry 
cycle index sum

$$
Z_{3}:=\frac{1}{2}\left(\sum_{k \geqslant 0}\left(2 \mathscr{D}\left(s_{2}\right)\right)^{k}\right) \mathscr{D}\left(s_{2}\right)=\frac{\mathscr{D}\left(s_{2}\right)}{2\left(1-2 \mathscr{D}\left(s_{2}\right)\right)} .
$$

Summing up, we obtain

$$
Z_{\left(\mathscr{B}^{\prime}\right)^{\iota} \mathscr{O}}=Z_{0}+\ldots+Z_{3}=\frac{1}{2}\left(\mathscr{D}\left(s_{1}\right)+\mathscr{D}\left(s_{2}\right) \frac{s_{1}}{s_{2}}+\frac{2 \mathscr{D}\left(s_{2}\right)^{2} \frac{s_{1}}{s_{2}}+\mathscr{D}\left(s_{2}\right)}{1-2 \mathscr{D}\left(s_{2}\right)}\right) .
$$

Using a computer algebra system, we may verify that this is identical to the expression given in Equation (55).

It follows from this description that the cycle index $\operatorname{sum} Z_{\left.(\mathscr{B})^{\bullet}\right)_{\mathscr{O}}}$ of bi-pointed twoconnected outerplanar graphs is given by

$$
Z_{\left(\mathscr{B}^{\prime \bullet}\right)^{\imath} \mathscr{O}}=Z_{0}^{\bullet}+Z_{1}^{\bullet}+Z_{2}^{\bullet}=\frac{1}{2}\left(\mathscr{D}^{\bullet}\left(s_{1}\right)+s_{1}\right)+Z_{1}+Z_{2}
$$

with $F^{\bullet}=s_{1} \frac{\partial F}{\partial s_{1}}$ for any series $F$. But what is important to compute $\mathbb{E}[\eta]$ is not just the series but its combinatorial interpretation, which is why we made the effort to derive these equations, rather than just recalling (55). For ease of notation, let us set $a=\tilde{\mathscr{A}}^{\omega}\left(\rho_{\mathscr{A}}\right)$ and $b=\tilde{A}^{\omega}\left(\rho_{\mathscr{A}}^{2}\right)$. In order to sample the Boltzmann distributed random graph $\mathrm{B}^{\prime \bullet}$, we may sample a symmetry from $\bigcup_{k \geqslant 1} \operatorname{Sym}\left(\mathscr{B}^{\prime \bullet}\right)[k]$ according to an $(a, b)$-Boltzmann distribution for $\operatorname{Sym}\left(\mathscr{B}^{\prime \bullet}\right)$ (that is, any symmetry $(B, \sigma)$ is attained with probability $\left.\frac{\iota_{\mathscr{O}}(B)}{|B| !} a^{\sigma_{1}} b^{\sigma_{2}} / Z_{(\mathscr{B})^{\iota} \mathscr{O}}(a, b)\right)$ and then forget about the automorphism. The decomposition that lead us to (61) allows us to do sample this random symmetry in a multi-step process.

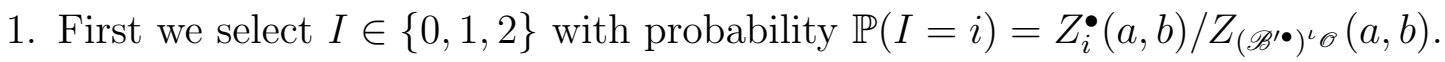

2. If $I=0$, we sample a random labelled graph $\mathrm{B}^{0}$ from $\bigcup_{k \geqslant 0}\left(\mathscr{B}^{\prime \bullet}\right)^{\iota \mathscr{O}}[k]$ with probability given by $\mathbb{P}\left(\mathrm{B}^{0}=B\right)=\frac{\iota_{\mathscr{O}}(|B|)}{|B| !} a^{|B|} /\left(\frac{1}{2} \mathscr{D} \bullet(a)+a\right)$ and equip it with the trivial automorphism.

3. If $I \in\{1,2\}$ then $Z_{i}^{\bullet}=Z_{i}$, as any symmetry constructed to form $Z_{i}$ has a unique fixed atom. We select a random symmetry $\left(\mathrm{B}^{I}, \sigma(I)\right)$ among all the symmetries constructed to sum up the cycle index sum $Z_{I}$ in (58) (in case $I=1$ ) or (59) (in case $I=2$ ), respectively, with probability

$$
\mathbb{P}\left(\left(\mathrm{B}^{I}, \sigma(I)\right)=(B, \sigma)\right)=\frac{\iota_{\mathscr{O}}(B)}{|B| !} a^{\sigma_{1}(I)} b^{\sigma_{2}(I)} / Z_{I}(a, b) .
$$

Let $\eta_{I}$ denote the distance between the $*$-vertex and the marked root in the random graph $\mathrm{B}^{I}$. This yields

$$
\mathbb{E}[\eta]=\frac{1}{Z_{\left(\mathscr{B}^{\prime \bullet}\right)^{\iota} \mathscr{O}}(a, b)}\left(\frac{1}{2}\left(a \mathscr{D}^{\prime}(a)+a\right) \mathbb{E}\left[\eta_{0}\right]+Z_{1}(a, b) \mathbb{E}\left[\eta_{1}\right]+Z_{2}(a, b) \mathbb{E}\left[\eta_{2}\right]\right) .
$$


Recall that we constructed the symmetries for $Z_{1}$ as in Figure 5 so that there exists always an edge between the $*$-vertex and the marked root. Hence $\eta_{1}=1$ is constant and so

$$
\mathbb{E}\left[\eta_{1}\right]=1 .
$$

As for the case $I=2$ (see the upper half of Figure 6 ), the distance $\eta_{2}$ is equal to the number of dissections attached to the root-face along one half of the symmetry axis. By the arguments that lead to Equation (59), it follows that $\eta \geqslant 2$ and for any $k \geqslant 2$ it holds $\mathbb{P}\left(\eta_{2}=k\right)=(2 \mathscr{D}(b))^{k} \frac{a}{4 b} / Z_{2}(a, b)$. This yields

$$
\mathbb{E}\left[\eta_{2}\right]=Z_{2}(a, b)^{-1} \sum_{k \geqslant 2} k(2 \mathscr{D}(b))^{k} \frac{a}{4 b}=\frac{2-2 \mathscr{D}(b)}{1-2 \mathscr{D}(b)} .
$$

Finally, consider the case $I=0$. Here we actually treat random labelled graphs, and we may build upon results obtained in this setting. It follows from the arguments that lead to Equation (57) that

$$
\mathbb{E}\left[\eta_{0}\right]=\frac{a}{\mathscr{D} \bullet(a)+a}+\frac{\mathscr{D} \bullet(a)}{\mathscr{D} \bullet(a)+a} \mathbb{E}\left[\eta_{0}^{\prime}\right]
$$

with $\mathscr{D}^{\bullet}(z)=z \mathscr{D}^{\prime}(z)$ and $\eta_{0}^{\prime}$ the distance between the $*$-vertex and the marked root in a random marked dissection from the class $\mathscr{D}^{\bullet}$ that assumes any marked dissection $D^{\bullet}$ with probability $\frac{a^{\left|D^{\bullet}\right|}}{\left|D^{\bullet}\right| !} / \mathscr{D} \bullet(a)$. Let us set $w:=\mathscr{D}(a)$. It follows from the proof of [30, Lem. 8.9], where calculations for marked dissection where carried out with different parameters, that there exist numbers $R, S>0$ such that

$$
\begin{aligned}
& \left(\begin{array}{ccc}
2 w^{4}-4 w^{3}+3 w-1 & -w^{3}+w^{2} & w^{3}-2 w^{2}+w \\
-w^{3}+w^{2} & 2 w^{4}-4 w^{3}+3 w-1 & w^{3}-2 w^{2}+w \\
-w^{2}+w & -w^{2}+w & 2 w^{4}-4 w^{3}+w^{2}+2 w-1
\end{array}\right)\left(\begin{array}{c}
\mathbb{E}\left[\eta_{0}^{\prime}\right] \\
R \\
S
\end{array}\right) \\
& =\left(\begin{array}{c}
2 w^{4}-4 w^{3}-w^{2}+3 w-1 \\
-w \\
-w^{2}
\end{array}\right) \text {. }
\end{aligned}
$$

This inhomogeneous system of linear equations (with the indeterminates $\mathbb{E}\left[\eta_{0}^{\prime}\right], R, S$ ) had a unique solution for the parameter considered in [30, Lem. 8.9], but we still have to check if this the case in our setting. The growth constant for unlabelled outerplanar graphs was approximated in [37, Sec. 3.1.3], [9, Sec. 4.2] by numerically solving truncated systems of equations, yielding $\rho_{\mathscr{A}} \approx 0.1332694, a \approx 0.1707560, b \approx 0.0180940, E_{u u}\left(\rho_{\mathscr{A}}, a\right) \approx 549.359$ and $E_{z}\left(\rho_{\mathscr{A}}, a\right) \approx 1.34975$. See [37, Sec. 3.1.3] for preciser estimates, that we used to carry out all following calculations. The determinate of the matrix in the system of linear equations evaluates to $\approx-0.00805 \neq 0$. Hence there exists a unique solution of the associated inhomogeneous system, yielding $\mathbb{E}\left[\eta_{0}^{\prime}\right]=\frac{8 w^{4}-16 w^{3}+4 w-1}{\left(4 w^{3}-6 w^{2}-2 w+1\right)(2 w-1)} \approx 5.435858$. This allows us to evaluate Equation (62), yielding $\mathbb{E}[\eta] \approx 5.038561$. Using Equation (54) we obtain $\mathbb{E}[\zeta] \approx 0.0534353$ and $\mathbb{V}[\xi] \approx 93.80631$. Hence Equation $(52)$ evaluates to

$$
c_{\omega_{\mathscr{O}}} \approx 0.9864689 \text {, }
$$

as we stated in Theorem 3 . 


\section{Acknowledgement}

I warmly thank the referee for the thorough reading and helpful remarks.

\section{A Species theory}

In this appendix, we summarize some aspects of combinatorial species used in our proofs following $[23,7,10]$. A thorough introduction to the subject is beyond the scope of this paper and we refer the reader to these sources.

\section{A.1 Weighted combinatorial species}

We are going to define combinatorial species with weights in the set $\mathbb{R}_{\geqslant 0}$ of non-negative real numbers. Such an object $\mathscr{F}^{\omega}$ may be described as follows. For any finite set $U$ the species $\mathscr{F}^{\omega}$ produces a finite set $\mathscr{F}[U]$ of $\mathscr{F}$-structures and a weight-map $\omega_{U}: \mathscr{F}[U] \rightarrow$ $\mathbb{R}_{\geqslant 0}$. Furthermore, for any bijection $\sigma: U \rightarrow V$ between finite sets the species $\mathscr{F}^{\omega}$ produces a transport function $\mathscr{F}[\sigma]: \mathscr{F}[U] \rightarrow \mathscr{F}[V]$, which must preserve the $\omega$-weights. In other words, the diagram

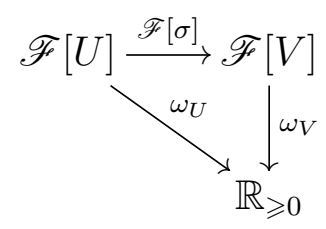

is required to commute. The bijections produced by a species are subject to functoriality conditions: the identity map $\mathrm{id}_{U}$ on a finite set $U$ gets mapped to the identity map $\mathscr{F}\left[\operatorname{id}_{U}\right]=\operatorname{id}_{\mathscr{F}[U]}$ on the set $\mathscr{F}[U]$. For any bijections $\sigma: U \rightarrow V$ and $\gamma: V \rightarrow W$ the diagram

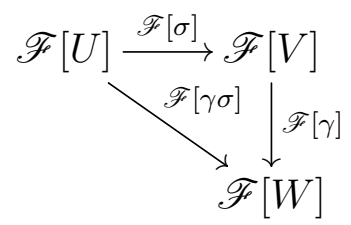

must commute. We further assume that $\mathscr{F}[U] \cap \mathscr{F}[V]=\emptyset$ whenever $U \neq V$. This is not much of a restriction, as we may always replace $\mathscr{F}[U]$ by $\{U\} \times \mathscr{F}[U]$ for all sets $U$, to make sure that it is satisfied. A reader familiar with category theory may without doubt recognize that combinatorial species are endo-functors of the groupoid of finite weighted sets and weight-preserving bijections. In particular, any concerns regarding set-theoretic aspects of the definition of combinatorial species may be dispersed by consulting any book on category theory, in particular the standard treaty [25].

Two weighted species $\mathscr{F}^{\omega}$ and $\mathscr{H}^{\nu}$ are isomorphic, denoted by $\mathscr{F}^{\omega} \simeq \mathscr{H}^{\nu}$, if there exists a family of weight-preserving bijections $\left(\alpha_{U}: \mathscr{F}[U] \rightarrow \mathscr{H}[U]\right)_{U}$ with $U$ ranging over all finite sets, such the following diagram commutes for each bijection $\sigma: U \rightarrow V$ of 
finite sets.

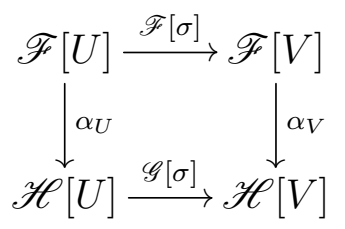

We say $\mathscr{H}^{\nu}$ is a subspecies of $\mathscr{F}^{\omega}$, if for each finite set $U$, any bijection $\sigma: U \rightarrow V$ and each $\mathscr{H}$-object $H \in \mathscr{H}^{\nu}$ it holds that $\mathscr{H}[U] \subset \mathscr{F}[U], \mathscr{H}[\sigma](H)=\mathscr{F}[\sigma](H)$ and $\nu_{U}(H)=\omega_{U}(H)$. We denote this by $\mathscr{H}^{\nu} \subset \mathscr{F}^{\omega}$. By abuse of notation, we will usually denote the weighting on both $\mathscr{H}$ and $\mathscr{F}$ by $\omega$.

It will be convenient to simply write $\omega(F)$ instead of $\omega_{U}(F)$ for the weight of a structure $F \in \mathscr{F}[U]$. If no weighting is specified explicitly for a species $\mathscr{F}$, we assume that all structures receive weight 1 . We refer to the set $U$ as the set of labels or atoms of the structure. For any $\mathscr{F}$-object $F \in \mathscr{F}[U]$ we let $|F|:=|U| \geqslant 0$ denote its size.

\section{A.2 Ordinary generating series and cycle index sums}

Given a finite set $U$, the symmetric group $\mathscr{S}_{U}$ operates on the set $U$ via

$$
\sigma . F=\mathscr{F}[\sigma](F)
$$

for all $F \in \mathscr{F}[U]$ and $\sigma \in \mathscr{S}_{U}$. Any bijection $\sigma$ with $\sigma . F=F$ is termed an automorphism of $F$. All $\mathscr{F}$-objects of an orbit $\tilde{F}$ have the same size and same $\omega$-weight, which we denote by $|\tilde{F}|$ and $\omega(\tilde{F})$. This yields the weighted set $\tilde{\mathscr{F}}[U]$ of orbits under this operation. Formally, an unlabelled $\mathscr{F}$-object is defined as an isomorphism class of $\mathscr{F}$-objects. We may also identify the unlabelled objects of a given size $n$ with the orbits of the action of the symmetric group on any $n$-sized set. By abuse of notation, we treat unlabelled objects as if they were regular $\mathscr{F}$-objects. The power series

$$
\tilde{\mathscr{F}}^{\omega}(z)=\sum_{\tilde{F}} \omega(\tilde{F}) z^{|\tilde{F}|}
$$

is the ordinary generating series of the species. Here the index ranges over all unlabelled $\mathscr{F}$-objects.

To any species $\mathscr{F}$ we may associate the corresponding functor $\operatorname{Sym}(\mathscr{F})$ of $\mathscr{F}$-symmetries such that

$$
\operatorname{Sym}(\mathscr{F})[U]=\left\{(F, \sigma) \mid F \in \mathscr{F}[U], \sigma \in \mathscr{S}_{U}, \sigma \cdot F=F\right\} .
$$

In other words, a symmetry is a pair of an $\mathscr{F}$-object and an automorphism. The transport along a bijection $\gamma: U \rightarrow V$ is given by

$$
\operatorname{Sym}(\mathscr{F})[\gamma](F, \sigma)=\left(\mathscr{F}[\gamma](F), \gamma \sigma \gamma^{-1}\right) .
$$

For any permutation $\sigma$ we let $\sigma_{i}$ denote its number of $i$-cycles. In particular, $\sigma_{1}$ counts the number of fixpoints. The cycle index series of a species $\mathscr{F}^{\omega}$ is defined as the formal power series

$$
Z_{\mathscr{F} \omega}\left(s_{1}, s_{2}, \ldots\right)=\sum_{k \geqslant 0} \sum_{(F, \sigma) \in \operatorname{Sym}(\mathscr{F})[k]} \frac{\omega(F)}{k !} s_{1}^{\sigma_{1}} \cdots s_{k}^{\sigma_{k}}
$$


in countably infinitely many indeterminates $\left(s_{i}\right)_{i \geqslant 1}$. Consider symmetries is useful, as it provides a way of counting orbits:

Lemma 12. For any finite set $U$ with $n$ elements and any unlabelled $\mathscr{F}$-object $\tilde{F} \in$ $\tilde{\mathscr{F}}[U]$ there are precisely $n !$ many symmetries $(F, \sigma) \in \operatorname{Sym}(\mathscr{F})[U]$ such that $F$ belongs to the orbit $\tilde{F}$. Hence there exists a weight-preserving 1 to $n$ ! relation between $\tilde{\mathscr{F}}[U]$ and $\operatorname{Sym}(\mathscr{F})[U]$. Consequently:

$$
\tilde{\mathscr{F}}^{\omega}(z)=Z_{\mathscr{F} \omega}\left(z, z^{2}, z^{3}, \ldots\right) .
$$

This standard result is explicit in Bergeron, Labelle and Leroux [7, Ch. 2.3].

\section{A.3 The cycle pointing operator}

For each finite set $U$ and permutation $\sigma \in \mathscr{S}_{U}$, the generated subgroup $\langle\sigma\rangle \subset \mathscr{S}_{U}$ operates canonically on $U$. The restriction of $\sigma$ to any single orbit of this operation is termed a cycle of $\sigma$. For any cycle $\tau$ we let its length $|\tau|$ be the number of elements of the corresponding orbit. The cycle-pointed species $\left(\mathscr{F}^{\circ}\right)^{\omega}$ associated to a species $\mathscr{F}^{\omega}$ is defined as follows. For each finite set $U$, the elements of the set $\mathscr{F}^{\circ}[U]$ are all pairs $(F, \tau)$ of an $\mathscr{F}$-structure $F$ and a cyclic permutation $\tau$ of some subset of $U$ such that there exists at least one automorphism $\sigma \in \mathscr{S}_{U}$ of $F$ having $\tau$ as one of its disjoint cycles. (Here we allow the case where $\tau$ is just a fixed-point of $\sigma$.) The transport along a bijection $\gamma: U \rightarrow V$ is defined by

$$
\mathscr{F}^{\circ}[\gamma](F, \tau)=\left(\mathscr{F}[\gamma](F), \gamma \tau \gamma^{-1}\right)
$$

The weighting of the cycle-pointed version is inherited from the original species by

$$
\omega(F, \tau)=\omega(F)
$$

The idea behind the cycle pointing operator is that it provides a way for counting objects up to symmetry.

Lemma 13. For any finite set $U$ with $n$ vertices there exists a weight-preserving 1 to $n$ correspondence between the set $\tilde{\mathscr{F}}[U]$ of orbits of $\mathscr{F}$-objects and the set $\tilde{\mathscr{F}}^{\circ}[U]$ of orbits of $\mathscr{F}^{\circ}$-objects.

This result has been proven in [10, Lemma 4] in the context of species without weightings, and the generalization to the weighted context is straight-forward. Lemma 13 shows that there is no difference in sampling a random $n$-sized unlabelled object with probability proportional to its weight from $\mathscr{F}$ and $\mathscr{F}^{\circ}$.

Any subspecies $\mathscr{H}^{\nu} \subset\left(\mathscr{F}^{\circ}\right)^{\omega}$ is termed cycle-pointed as well. A natural example is the subspecies $\left(\mathscr{F}^{\circledast}\right)^{\omega} \subset\left(\mathscr{F}^{\circ}\right)^{\omega}$ of symmetrically cycle-pointed objects for which the length of the marked cycle of each object is required to be at least 2 . For any finite set $U$ we let $\operatorname{RSym}(\mathscr{H})[U]$ denote the set of all tuples $(H, \sigma, \tau, v)$ with $(H, \tau) \in \mathscr{H}[U], \sigma$ an automorphism of $H$ having $\tau$ as one of its disjoint cycles, and $v \in U$ an atom of the cycle 
$\tau$. In order to keep track of the length of the marked cycle, cycle-pointed species receive an extended version of the cycle index sum

$$
\bar{Z}_{\mathscr{H}^{\nu}}\left(s_{1}, t_{1} ; s_{2}, t_{2} ; \ldots\right)=\sum_{k \geqslant 0} \frac{1}{k !} \sum_{(H, \sigma, \tau, v) \in \operatorname{RSym}(\mathscr{H})[k]} \nu(H) \frac{t_{|\tau|}}{s_{|\tau|}} s_{1}^{\sigma_{1}} s_{2}^{\sigma_{2}} \ldots s_{k}^{\sigma_{k}} .
$$

Lemma 13 readily implies that

$$
\tilde{\mathscr{H}}^{\nu}(z)=\bar{Z}_{\mathscr{H}}\left(z, z ; z^{2}, z^{2} ; \ldots\right) .
$$

\section{A.4 Further operators}

There are various standard ways to combine given species (cycle-pointed or not) to form new ones. We briefly recall some notation and relevant facts, but refer the reader to the literature [7, Section 2.3] and [10] for a thorough description of these constructions. Throughout we let $\mathscr{F}^{\omega}$ and $\mathscr{G}^{\nu}$ denote weighted species.

\section{A.4.1 Constructions without cycle-pointing}

If $\mathscr{G}^{\nu}[\emptyset]=\emptyset$ then we may form the composition or substitution $\mathscr{F}^{\omega} \circ \mathscr{G}^{\nu}$. It is a weighted species that describes partitions of finite sets, where each partition class is endowed with a $\mathscr{G}$-structure, and the collection of partition classes carries an $\mathscr{F}$-structure. The weight of such a composite structure is the product of weights of its $\mathscr{F}^{\omega}{ }^{\omega}$-structure and $\mathscr{G}^{\nu}$-structures. The cycle index sum of the substitution is given by

$$
Z_{\mathscr{F} \omega_{\circ} \mathscr{G \nu}}\left(s_{1}, s_{2}, \ldots\right)=Z_{\mathscr{F} \omega}\left(Z_{\mathscr{G} \nu}\left(s_{1}, s_{2}, \ldots\right), Z_{\mathscr{G} \nu^{2}}\left(s_{2}, s_{4}, \ldots\right), Z_{\mathscr{G} \nu^{3}}\left(s_{3}, s_{6}, \ldots\right), \ldots\right) .
$$

Here $\nu^{i}$ denotes the weighting that assigns to each $\mathscr{G}$-object $G$ the weight $\nu^{i}(G)=\nu(G)^{i}$. See for example [23, Theorem 3 and Section 6] or [7, Proposition 11 of Section 2.3] for details.

The product $\mathscr{F}^{\omega} \cdot \mathscr{G}^{\nu}$ describes ordered pairs of an $\mathscr{F}^{\omega}$ and an $\mathscr{G}^{\nu}$ structure. The weight of such a structure is the product of weights of its components. The cycle index sum of the product satisfies $Z_{\mathscr{F} \omega . \mathscr{G} \mu}=Z_{\mathscr{F} \omega} Z_{\mathscr{G} \nu}$. The sum $\mathscr{F}^{\omega}+\mathscr{G}^{\nu}$ describes the disjoint union of the two species, that canonically extends to weights and transport functions. The cycle index sum of the sum satisfies $Z_{\mathscr{F} \omega+\mathscr{G} \nu}=Z_{\mathscr{F} \omega}+Z_{\mathscr{G} \nu}$. It is straight-forward to generalize this concept to sums of countably many species subject to the summability constraint that in total only finitely many unlabelled objects of any fixed size are present. The derived species $\left(\mathscr{F}^{\prime}\right)^{\omega}$ describes $\mathscr{F}^{\omega}$-objects where one atom is marked and no longer contributes to the total size. Its cycle index series is given by $Z_{\left(\mathscr{F}^{\prime}\right) \omega}\left(s_{1}, s_{2}, \ldots\right)=\frac{\partial}{\partial s_{1}} Z_{\mathscr{F} \omega}\left(s_{1}, s_{2}, \ldots\right)$. Similar to the derived species, the pointed species $\mathscr{F}^{\bullet}$ is given by $\mathscr{F}^{\bullet}=\mathscr{F}^{\prime} \cdot \mathscr{X}$ with $\mathscr{X}$ is the species having a single object of size 1 and weight 1 .

\section{A.4.2 Constructions for cycle-pointed species}

It the species $\mathscr{F}^{\omega} \subset\left(\mathscr{H}^{\circ}\right)^{\omega}$ is cycle-pointed and $\mathscr{G}[\emptyset]=\emptyset$ we may form the cycle-pointed substitution $\mathscr{F}^{\omega} \odot \mathscr{G}^{\nu}$ as follows. Given an $\left(\mathscr{H} \circ \mathscr{G}^{\circ}\right)^{\circ}$-structure $\left(\left(H,\left(G_{Q}\right)_{Q \in \pi}\right), \tau\right)$, there 
must be an automorphism $\sigma$ having $\tau$ as one of its cycles. Let $\bar{\sigma}: \pi \rightarrow \pi, Q \mapsto \sigma(Q)$ denote the corresponding induced map on the partition. For each atom $v$ of the cycle $\tau$ let $Q(v) \in \pi$ denote the unique partition class to which it belongs. Clearly it must hold that $\bar{\sigma}(Q(v))=Q(\tau(v))$. Hence $\bar{\sigma}$ restricted to the set $\{Q(v) \mid v \in \tau\}$ forms a cycle $\bar{\tau}$. This makes $(H, \bar{\tau}) \in \mathscr{H}^{\circ}[\pi]$ a cycle-pointed $\mathscr{H}$-structure, that is called the core structure. If the core structure belongs to the subset $\mathscr{F}[\pi] \subset \mathscr{H}^{\circ}[\pi]$, then we say $\left(\left(H,\left(G_{Q}\right)_{Q \in \pi}\right), \tau\right)$ belongs to the cycle-pointed substitution of $\mathscr{F}^{\omega}$ with $\mathscr{G}^{\nu}$. This defines a subspecies $\mathscr{F}^{\omega} \odot \mathscr{G}^{\nu} \subset\left(\mathscr{H}^{\omega} \circ \mathscr{G}^{\nu}\right)^{\circ}$, and the weighting on $\mathscr{F}^{\omega} \odot \mathscr{G}^{\nu}$ is inherited from $\left(\mathscr{H}^{\omega} \circ \mathscr{G}^{\nu}\right)^{\circ}$. By [10, Prop. 18], the extended cycle index sum of the cycle-pointed substitution is given by

$$
\bar{Z}_{\mathscr{F} \omega \odot \mathscr{G} \nu}\left(s_{1}, t_{1} ; s_{2}, t_{2} ; \ldots\right)=\bar{Z}_{\mathscr{F} \omega}\left(g_{1}, \bar{g}_{1} ; g_{2}, \bar{g}_{2} ; \ldots\right)
$$

with $g_{i}=Z_{\mathscr{G} \nu^{i}}\left(s_{i}, s_{2 i}, s_{3 i}, \ldots\right)$ and $\bar{g}_{i}=\bar{Z}_{(\mathscr{G} \circ)^{\nu}}\left(s_{i}, t_{i} ; s_{2 i}, t_{2 i} ; s_{3 i}, t_{3 i} ; \ldots\right)$. To be precise, [10, Prop. 18] states this equality for species without weights, but the generalization to the weighted context is straight-forward.

With the species $\mathscr{F}^{\omega}$ being cycle-pointed, the product $\mathscr{G}^{\nu} \cdot \mathscr{F}^{\omega}$ may also be interpreted as a cycle-pointed species $\mathscr{G}^{\nu} \star \mathscr{F}^{\omega}$, since the marked cycle of the $\mathscr{F}$-structure is also a cycle of some automorphism of the $\mathscr{G} \cdot \mathscr{F}$-structure. The corresponding extended cycle index sum is given by $\bar{Z}_{\mathscr{G}^{\nu} \mathscr{F}^{\omega}}=Z_{\mathscr{G} \nu} \bar{Z}_{\mathscr{F} \omega}$. Likewise, if $\mathscr{F}^{\omega}$ and $\mathscr{G}^{\nu}$ are both cycle-pointed, then so is their sum $\mathscr{F}^{\omega}+\mathscr{G}^{\nu}$ and the extended cycle index sum satisfies $\bar{Z}_{\mathscr{F} \omega+\mathscr{G} \nu}=\bar{Z}_{\mathscr{F} \omega}+\bar{Z}_{\mathscr{G} \nu}$. See [10] for details.

\section{A.5 Associative laws}

There are natural associative laws for the sum, product and substitution operations of the form

$$
\left(\mathscr{F}^{\omega} \mu \mathscr{G}^{\nu}\right) \mu \mathscr{H}^{\kappa} \simeq \mathscr{F}^{\omega} \mu\left(\mathscr{G}^{\nu} \mu \mathscr{H}^{\kappa}\right)
$$

for $\mu \in\{+, \cdot, \circ\}$, that ensure that regardless how we put the parentheses, the results are always isomorphic as species. Even more, there are natural choices of isomorphisms in (68) such that regardless in which order we successively apply the associative law to change from one parenthesization to another, the resulting concatenations of isomorphisms are always identical. It is for this strong form of associativity up to canonical isomorphism that we may drop the parentheses without any hazard [25, Ch. VII]. The inclined reader may consult [23, Ch. 7] for further details.

\section{References}

[1] R. Abraham, J.-F. Delmas, and P. Hoscheit. A note on the Gromov-HausdorffProkhorov distance between (locally) compact metric measure spaces. Electron. J. Probab., 18:no. 14, 21, 2013. 
[2] L. Addario-Berry, O. Angel, G. Chapuy, É. Fusy, and C. Goldschmidt. Voronoi tessellations in the CRT and continuum random maps of finite excess. Submitted, 2017.

[3] D. Aldous. The continuum random tree. I. Ann. Probab., 19(1):1-28, 1991.

[4] D. Aldous. The continuum random tree. II. An overview. In Stochastic analysis (Durham, 1990), volume 167 of London Math. Soc. Lecture Note Ser., pages 23-70. Cambridge Univ. Press, Cambridge, 1991.

[5] D. Aldous. The continuum random tree. III. Ann. Probab., 21(1):248-289, 1993.

[6] J. P. Bell, S. N. Burris, and K. A. Yeats. Counting rooted trees: the universal law $t(n) \sim C \rho^{-n} n^{-3 / 2}$. Electron. J. Combin., 13(1):\#R63, 2006.

[7] F. Bergeron, G. Labelle, and P. Leroux. Combinatorial species and tree-like structures, volume 67 of Encyclopedia of Mathematics and its Applications. Cambridge University Press, Cambridge, 1998.

[8] O. Bodini and Y. Ponty. Multi-dimensional Boltzmann sampling of languages. In Proceeding of the 21st international meeting on probabilistic, combinatorial, and asymptotic methods in the analysis of algorithms (AofA'10), Vienna, Austria, June 28 July 2, 2010, pages 49-64. Nancy: The Association. Discrete Mathematics \& Theoretical Computer Science (DMTCS), 2010.

[9] M. Bodirsky, É. Fusy, M. Kang, and S. Vigerske. Enumeration and asymptotic properties of unlabeled outerplanar graphs. Electron. J. Combin., 14(1):\#R66, 2007.

[10] M. Bodirsky, É. Fusy, M. Kang, and S. Vigerske. Boltzmann samplers, Pólya theory, and cycle pointing. SIAM J. Comput., 40(3):721-769, 2011.

[11] N. Broutin and P. Flajolet. The distribution of height and diameter in random non-plane binary trees. Random Structures Algorithms, 41(2):215-252, 2012.

[12] A. Caraceni. The scaling limit of random outerplanar maps. Ann. Inst. H. Poincaré Probab. Statist., 52(4):1667-1686, 112016.

[13] G. Chapuy and G. Perarnau. Local convergence and stability of tight bridge-addable classes. Can. J. Math., 72(3):563-601, 2020.

[14] R. Diestel. Graph theory, volume 173 of Graduate Texts in Mathematics. Springer, Heidelberg, fourth edition, 2010.

[15] M. Drmota, É. Fusy, M. Kang, V. Kraus, and J. Rué. Asymptotic study of subcritical graph classes. SIAM J. Discrete Math., 25(4):1615-1651, 2011.

[16] M. Drmota and M. Noy. Extremal parameters in sub-critical graph classes. In ANALCO13-Meeting on Analytic Algorithmics and Combinatorics, pages 1-7. SIAM, Philadelphia, PA, 2013.

[17] M. Drmota, L. Ramos, and J. Rué. Subgraph statistics in subcritical graph classes. Random Struct. Algorithms, 51(4):631-673, 2017.

[18] R. T. Durrett and D. L. Iglehart. Functionals of Brownian meander and Brownian excursion. Ann. Probab., 5:130-135, 1977.

[19] P. Flajolet and R. Sedgewick. Analytic combinatorics. Cambridge University Press, Cambridge, 2009. 
[20] A. Georgakopoulos and S. Wagner. Limits of subcritical random graphs and random graphs with excluded minors. arXiv:1512.03572, Dec. 2015.

[21] O. Giménez and M. Noy. Asymptotic enumeration and limit laws of planar graphs. J. Amer. Math. Soc., 22(2):309-329, 2009.

[22] B. Gittenberger, E. Y. Jin, and M. Wallner. On the shape of random Pólya structures. Discrete Mathematics, 341(4):896 - 911, 2018.

[23] A. Joyal. Une théorie combinatoire des séries formelles. Adv. in Math., 42(1):1-82, 1981.

[24] M. Krivelevich, K. Panagiotou, M. Penrose, and C. McDiarmid. Random graphs, geometry and asymptotic structure, volume 84 of London Mathematical Society Student Texts. Cambridge University Press, Cambridge, 2016. Edited by Nikolaos Fountoulakis and Dan Hefetz.

[25] S. Mac Lane. Categories for the working mathematician, volume 5 of Graduate Texts in Mathematics. Springer-Verlag, New York, second edition, 1998.

[26] C. McDiarmid. Random graphs from a minor-closed class. Combin. Probab. Comput., 18(4):583-599, 2009.

[27] C. McDiarmid and A. Scott. Random graphs from a block-stable class. European Journal of Combinatorics, 58:96 - 106, 2016.

[28] G. Miermont. Tessellations of random maps of arbitrary genus. Ann. Sci. Éc. Norm. Supér. (4), 42(5):725-781, 2009.

[29] R. Otter. The number of trees. Ann. of Math. (2), 49:583-599, 1948.

[30] K. Panagiotou, B. Stufler, and K. Weller. Scaling limits of random graphs from subcritical classes. Ann. Probab., 44(5):3291-3334, 2016.

[31] B. Stufler. Scaling limits of random outerplanar maps with independent link-weights. Ann. Inst. H. Poincaré Probab. Statist., 53(2):900-915, 052017.

[32] B. Stufler. Gibbs partitions: The convergent case. Random Structures \&6 Algorithms, 53(3):537-558, 2018.

[33] B. Stufler. Random enriched trees with applications to random graphs. Electronic Journal of Combinatorics, 25(3), \#P3.11, 2018.

[34] B. Stufler. The continuum random tree is the scaling limit of unlabeled unrooted trees. Random Structures \& Algorithms, 55(2):496-528, 2019.

[35] B. Stufler. Local convergence of random planar graphs. arXiv:1908.04850, Aug. 2019.

[36] B. Stufler. Unlabelled Gibbs partitions. Combinatorics, Probability and Computing, 29(2):293-309, 2020.

[37] S. Vigerske. Asymptotic enumeration of unlabelled outerplanar graphs. Diploma Thesis, Humboldt-Universität Berlin, 2005.

[38] M. Wang. Height and diameter of Brownian tree. Electron. Commun. Probab., 20:15, 2015. Id/No 88. 